\title{
Spectral Properties of the Ruelle Operator on the Walters Class over Compact Spaces
}

\author{
Leandro Cioletti and Eduardo A. Silva
}

February 12, 2018

\begin{abstract}
Recently the Ruelle-Perron-Fröbenius theorem was proved for Hölder potentials defined on the symbolic space $\Omega=M^{\mathbb{N}}$, where (the alphabet) $M$ is any compact metric space. In this paper, we extend this theorem to the Walters space $W(\Omega)$, in similar general alphabets. We also describe in detail an abstract procedure to obtain the Fréchetanalyticity of the Ruelle operator under quite general conditions and we apply this result to prove the analytic dependence of this operator on both Walters and Hölder spaces. The analyticity of the pressure functional on Hölder spaces is established. An exponential decay of the correlations is shown when the Ruelle operator has the spectral gap property.

A new (and natural) family of Walters potentials (on a finite alphabet derived from the Ising model) not having an exponential decay of the correlations is presented. Because of the lack of exponential decay, for such potentials we have the absence of the spectral gap for the Ruelle operator. The key idea to prove the lack of exponential decay of the correlations are the Griffiths-Kelly-Sherman inequalities.
\end{abstract}

Key-words: Thermodynamic formalism, Ruelle operator, one-dimensional lattice, Analyticity of Pressure, Spectral Gap.

MSC2010: 37A60, 37A50, 82B05.

\section{Introduction}

The Ruelle-Perron-Fröbenius theorem is one of the most important results in modern thermodynamic formalism. Nowadays the Ruelle operator have become a standard tool in many different areas in dynamical systems and other branches of Mathematics and Mathematical Physics. The literature about Ruelle-Perron-Fröbenius theorem is vast, the following is a partial list of books and papers on this subject [2, 6, 14, 15, 20, 32, 34, 42].

The classical thermodynamic formalism was in its origin developed in the Bernoulli space $M^{\mathbb{N}}$, with $M$ being a finite alphabet, see [32]. This assumption on $M$ allows one conjugates, using a Markov partitions, the shift maps on the Bernoulli space with uniform hyperbolic maps in differentiable manifolds. 
The Ruelle operator formalism is also proved useful in the multifractal analysis context. Bowen in the seminal work [7] established a relationship between Hausdorff dimension of certain fractal sets and topological pressure in the context of conformal dynamics in one dimension. This technique is known as Bowen's equation and in subsequent works it was extended by Manning and McCluskey for dynamical systems on surfaces aiming to compute fractal dimension of horseshoes, see for more details [7, 30, 31] and also the introductory texts [3, 33].

The motivation to consider more general alphabets from dynamical system point of view is given, for example, in [36, 38] where proposed models with infinite alphabet $M=\mathbb{N}$ is used to describe non-uniformly hyperbolic maps, for example, the Manneville-Pomeau maps. In Classical Statistical Mechanics uncountable alphabets shows up, for example, in the so-called $O(n)$ models with $n \geq 2$. In these models the alphabet is $\mathbb{S}^{n-1}$ the unit sphere in the Euclidean space $\mathbb{R}^{n}$, for details see [17]. Unbounded alphabets as general standard Borel spaces, which includes compact and non-compact, are considered in details in [21. We should mention that ergodic optimization problems are also being considered in infinite countable alphabets, see [4, 11, 19, 36].

Walters work 44 marked the beginning of three decades of great activity in thermodynamic formalism where less regular than Hölder potentials were considered. This class of potentials is called Walters class or alternatively Walters space. A rather complete theory in Walters space were developed for finite and countable alphabets, see [11. In the Walters paper, the dynamical system is supposed to be defined on a class of compact sets, expansive and mixing and the potentials can be any positive summable variation function.

The aim of this work is to extend some results obtained in [44] as the Ruelle-Perron-Fröbenius theorem, the analytic dependence the Ruelle operator with respect to the potential, spectral properties of this operator and some consequences of either presence or absence of the spectral gap, as for example the pressure analyticity. The main difficulty in carrying out the construction of the Ruelle operator for uncountable alphabets is overcome by the introduction of what we call an $a$-priori probability measure on $M$, which is a common strategy in the theory of general DLR-Gibbs measures.

This paper is organized as follows. In Section 2, we prove under quite general conditions the analyticity of the Ruelle operator and its dual with respect to the potential $f$. The result is more general, in fact we show that if the Ruelle operator $\mathscr{L}_{f}$ leaves invariant a Banach algebra $\mathcal{K} \subset C(\Omega)$ for all $f \in \mathcal{K}$, then the maps $\mathcal{K} \ni f \rightarrow \mathscr{L}_{f}, \mathcal{K}^{*} \ni f \rightarrow \mathscr{L}_{f}^{*}$ are analytic. We use this result in Section 5 with $\mathcal{K}=C^{\gamma}(\Omega)$ to obtain the analyticity of the topological pressure $P: C^{\gamma}(\Omega) \rightarrow \mathbb{R}$, which extends the analogous results known in finite/discrete alphabets. We shall remark that even for discrete case, despite this being a folkloric result we were not able to find its rigorous proof in the literature.

Section 3 deals with the Walters space, notation $W(\Omega)$. In the discrete setting there are several known equivalent characterizations of the Walters condition, however in a more general setting as compact metric spaces, things are more subtle. We show that the natural generalization of the two most popular characterizations of the Walters class are not equivalent when the alphabet is uncountable. We give an explicit example illustrating this fact. We introduce what we call weak and strong Walters conditions. Finally a generalization of [44] is proved for general compact alphabets. We remark that this theorem is also a non-trivial generalization of 
one the main theorems in [1, 27] where the Ruelle operator on uncountable alphabet are taken into account.

In Section 7 we introduce a new family of potentials for which the Ruelle operator has absence of the spectral gap. This section is heavily based on the ideas borrowed from Statistical Mechanics and the Griffiths-Kelly-Sherman (GKS) inequalities, [24, 25, 26, 23]. For the convenience of the reader we precisely stated all the theorems we need only in the needed generality but we provide its classical references where general settings are presented. Some Ising model routine computations are presented in details in order to make our exposition self-contained for non-specialists in Statistical Mechanics. These potentials belong to a infinite dimensional linear subspace of $C(\Omega, \mathbb{R})$ whose intersection with the Walters space is an infinite dimensional linear subspace not contained in the Hölder space. On that space Dobrushin in [10] by using estimates of mean value of exponential functionals of random processes and latter Cassandro and Olivieri [8] employing a renormalization group idea together with the cluster expansion proved analyticity of the pressure. It worth mention that the subexponential decay obtained in Section 5 can not be recovered from the seminal Sarig's work [37] about subexponential decay of correlations and neither from the improvement provided by Gouëzel in [22]. The examples presented in Section 5 can shed some light in potential applications of the GKS inequalities to study the absence of the spectral gap in other situations.

\section{Basic Definitions}

In this section we setup the notation and present some preliminaries results. Let $M=(M, d)$ be a compact metric space, equipped with a Borel probability measure $\mu: \mathscr{B}(M) \rightarrow[0,1]$ having the whole space $M$ as its support. We shall denote by $\Omega=M^{\mathbb{N}}$ the set of all sequences $x=\left(x_{1}, x_{2}, \ldots\right)$, where $x_{i} \in M$, for all $i \in \mathbb{N}$. We denote by $\sigma: \Omega \rightarrow \Omega$ the left shift mapping which is given by $\sigma\left(x_{1}, x_{2}, \ldots\right)=\left(x_{2}, x_{3}, \ldots\right)$. We consider the metric $d_{\Omega}$ on $\Omega$ given by

$$
d_{\Omega}(x, y)=\sum_{n=1}^{\infty} \frac{1}{2^{n}} d\left(x_{n}, y_{n}\right)
$$

The metric $d_{\Omega}$ induces the product topology and therefore follows from the Tychnoff Theorem that $\left(\Omega, d_{\Omega}\right)$ is a compact metric space. The space of all the continuous real functions $C(\Omega, \mathbb{R})$ is denoted simply by $C(\Omega)$. For any fixed $0 \leq \gamma \leq 1$ we denote by $C^{\gamma}(\Omega)$ the space of all $\gamma$-Hölder continuous functions, i.e, the set of all functions $f: \Omega \rightarrow \mathbb{R}$ satisfying

$$
\operatorname{Hol}(f)=\sup _{x, y \in \Omega: x \neq y} \frac{|f(x)-f(y)|}{d_{\Omega}(x, y)^{\gamma}}<+\infty .
$$

We equip $C^{\gamma}(\Omega), 0 \leq \gamma \leq 1$ with the norm $\|\cdot\|_{\gamma}$ which is defined for $\gamma=0$ by $\|f\|_{0}=$ $\sup _{x \in \Omega}|f(x)|$ and for $0<\gamma \leq 1$ by $\|f\|_{\gamma}=\|f\|_{0}+\operatorname{Hol}(f)$. We recall that $\left(C^{\gamma}(\Omega),\|\cdot\|_{\gamma}\right)$ is a Banach space for any $0 \leq \gamma \leq 1$.

Our potentials will be elements of $C(\Omega)$ and in order to have a well defined Ruelle operator when $(M, d)$ is a general compact metric space we need to consider an a priori measure which is 
simply a Borel probability measure $\mu: \mathscr{B}(M) \rightarrow \mathbb{R}$, where $\mathscr{B}(M)$ denotes the Borel $\sigma$-algebra of $M$. For many of the most popular choices of an uncountable space $M$ there is a natural $a$ priori measure $\mu$. Throughout this paper the a priori measure $\mu$ is supposed to have the whole space $M$ as its support. The Ruelle operator $\mathscr{L}_{f}: C^{\gamma}(\Omega) \rightarrow C^{\gamma}(\Omega)$ is the mapping sending $\varphi \mapsto \mathscr{L}_{f}(\varphi)$ which is defined for any $x \in \Omega$ by the expression

$$
\mathscr{L}_{f}(\varphi)(x)=\int_{M} e^{f(a x)} \varphi(a x) d \mu(a)
$$

where $a x$ denotes the sequence $a x=\left(a, x_{1}, x_{2}, \ldots\right) \in \Omega$.

This operator is a generalization of the classical Ruelle operator and has been appeared lately in the Thermodynamic Formalism literature, see for example [1, 27, 39]. The classical Ruelle operator can be recovered on this setting by considering $M=\{0,1, \ldots, n\}$ and the $a$ priori $\mu$ as the counting measure. Our starting point is the following theorem.

Theorem 2.1 (Ruelle-Perron-Fröbenius). Let $(M, d)$ be a compact metric space, $\mu$ a Borel probability measure of full support on $M$ and $f$ be a potential in $C^{\gamma}(\Omega)$, where $0<\gamma<1$. Then $\mathscr{L}_{f}: C^{\gamma}(\Omega) \rightarrow C^{\gamma}(\Omega)$ have a simple positive eigenvalue of maximal modulus $\lambda_{f}$, and there are a strictly positive function $h_{f}$ and a Borel probability measure $\nu_{f}$ on $\Omega$ such that,

i) the remainder of the spectrum of $\mathscr{L}_{f}: C^{\gamma}(\Omega) \rightarrow C^{\gamma}(\Omega)$ is contained in a disc of radius strictly smaller then $\lambda_{f}$;

ii) for all continuous functions $\varphi \in C(\Omega)$ we have

$$
\lim _{n \rightarrow \infty}\left\|\lambda_{f}^{-n} \mathscr{L}_{f}^{n} \varphi-h_{f} \int_{\Omega} \varphi d \nu_{f}\right\|_{0}=0
$$

Proof. See [1] for the case $M=S^{1}$ and [27] for more general compact metric spaces.

Remark 2.2. Strictly speaking, in [1] and [27] the item (ii) above was proved only for Hölder continuous potentials. However this is enough since the space of the Hölder continuous potentials is dense in $\left(C(\Omega),\|\cdot\|_{0}\right)$. Therefore a straightforward computation shows that the convergence on the item (ii) holds for all $\varphi \in C(\Omega)$. The denseness of $C^{\gamma}(\Omega), 0<\gamma<1$ in $C(\Omega)$ is a consequence of the Stone Weierstrass Theorem. Indeed, $C^{\gamma}(\Omega)$ is an algebra of functions containing all the constant functions and if $x \neq y \in \Omega$, then the function $f$ given by $f(y)=$ $d_{\Omega}(y, x)^{\gamma}$, separates $x$ and $y$ and $f \in C^{\gamma}(\Omega)$. Since $\Omega$ is compact the result follows.

Following [1, 27] we define the entropy of a shift invariant measure $\nu \in \mathcal{M}_{\sigma}$ and the pressure of the potential $f$, respectively, as follows

$$
h(\nu)=\inf _{f \in C^{\gamma}(\Omega)}\left\{-\int_{\Omega} f d \nu+\log \lambda_{f}\right\} \quad \text { and } \quad P(f)=\sup _{\nu \in \mathcal{M}_{\sigma}}\left\{h(\nu)+\int_{\Omega} f d \nu\right\} .
$$

Proposition 2.3. For each $f \in C^{\gamma}(\Omega)$ we have for all $x \in \Omega$ that

$$
P(f)=\lim _{n \rightarrow \infty} \frac{1}{n} \log \left[\mathscr{L}_{f}^{n}(1)(x)\right]=\log \lambda_{f} .
$$


Proof. See [27] Corollary 1.

Another important property of the Ruelle operator is its analytic dependence (in the Fréchet's sense) with respect to the potential. The lemma below state it precisely.

Lemma 2.4. The map $\Theta: C^{\gamma}(\Omega) \rightarrow L\left(C^{\gamma}(\Omega), C^{\gamma}(\Omega)\right)$ sending $f \in C^{\gamma}(\Omega)$ to the Ruelle operator $\mathscr{L}_{f}$ associated to the potential $f$, is an analytic map.

Proof. See [39] Theorem 3.5.

One of the aims of this work is to extend the previous mentioned results to potentials on the Walters space $W(\Omega)$ (to be defined in the next section), where $\Omega$ is the infinite Cartesian product of a general compact metric space $(M, d)$.

We shall prove that the Ruelle operator and its dual depends analytically on the potential in $C^{\gamma}(\Omega)$ and then derive the analyticity of the pressure. In order to formulate these results using an unified setting, we need to introduce some additional notation. Let $\mathcal{K} \subset C(\Omega)$ be an arbitrary linear subspace of $C(\Omega)$, endowed with a norm $\|\cdot\|$. We use the notation $\mathcal{K}^{*}$ to denote the topological dual of $(\mathcal{K},\|\cdot\|)$. As usual, we define the norm of an element $\phi \in \mathcal{K}^{*}$ by $\|\phi\|_{*}=\sup \{|\phi(f)|: f \in \mathcal{K}$ and $\|f\|=1\}$. To lighten the notation, the space $L(\mathcal{K}, \mathcal{K})$ of the all continuous (strong topology) linear operators acting on $\mathcal{K}$ will be denote by $V \equiv L(\mathcal{K}, \mathcal{K})$.

Definition 2.5. Let $\mathcal{K} \subset C(\Omega)$ be a linear subspace. We say that $\mathcal{K}$ is invariant for the Ruelle operator, if for all $f \in \mathcal{K}$ we have $\mathscr{L}_{f} \mathcal{K} \subset \mathcal{K}$.

The central examples of invariant subspaces for the Ruelle operator appearing here are the spaces $C^{\gamma}(\Omega), 0<\gamma \leq 1$, and the Walters Space $W(\Omega)$.

The next proposition plays a key role in the study of the analyticity of the pressure. Its first statement is a simple generalization of the Theorem 3.5 in [39], which is presented here for the reader's convenience. The second one follows from the first after some work.

Proposition 2.6. Suppose that $\mathcal{K} \subset C(\Omega)$ is equipped with a norm $\|\cdot\|$ so that $(K,\|\cdot\|)$ is a Banach Algebra, $\mathcal{K}$ is invariant for the Ruelle operator and for any $f \in \mathcal{K}$ assume that $\mathscr{L}_{f} \in V$. Then both mappings $\Theta$ and $\Theta^{*}$ given by

$$
\mathcal{K} \ni f \mapsto \mathscr{L}_{f} \in L(\mathcal{K}, \mathcal{K}) \quad \text { and } \quad \mathcal{K} \ni f \mapsto \mathscr{L}_{f}^{*} \in L\left(\mathcal{K}^{*}, \mathcal{K}^{*}\right)
$$

define analytic functions.

Before proving the above proposition we state an immediate corollary of it which is an important tool to obtain the analyticity of the pressure functional.

Corollary 2.7. For each $0<\gamma \leq 1$, both mappings

$$
C^{\gamma}(\Omega) \ni f \mapsto \mathscr{L}_{f} \in L\left(C^{\gamma}(\Omega), C^{\gamma}(\Omega)\right) \quad \text { and } \quad C^{\gamma}(\Omega) \ni f \mapsto \mathscr{L}_{f}^{*} \in L\left(C^{\gamma}(\Omega)^{*}, C^{\gamma}(\Omega)^{*}\right)
$$

define analytic maps. 
Proof. Notice that the subspace $C^{\gamma}(\Omega)$ is invariant for the Ruelle operator and $\left(C^{\gamma}(\Omega),\|\cdot\|_{\gamma}\right)$ is a Banach Algebra (see [39]), so we are done.

\section{Proof of the Proposition 2.6.}

We first prove the analyticity of $\Theta$. Given $f, h \in \mathcal{K}$ and $\varphi \in C(\Omega)$ we have for any $x \in \Omega$ the following equality

$$
\begin{aligned}
\Theta(f+h)(\varphi)(x)-\Theta(f)(\varphi)(x) & =\mathscr{L}_{f+h}(\varphi)(x)-\mathscr{L}_{f}(\varphi)(x) \\
& =\int_{M} e^{f(a x)+h(a x)} \varphi(a x) d \mu(a)-\int_{M} e^{f(a x)} \varphi(a x) d \mu(a) \\
& =\int_{M} e^{f(a x)} \varphi(a x)\left(e^{h(a x)}-1\right) d \mu(a) \\
& =\int_{M}\left(e^{f(a x)} \varphi(a x) \sum_{n=1}^{\infty} \frac{[h(a x)]^{n}}{n !}\right) d \mu(a) .
\end{aligned}
$$

As long as the Fubini Theorem applies we get

$$
\Theta(f+h)(\varphi)(x)-\Theta(f)(h)(x)=\sum_{n=1}^{\infty} \frac{1}{n !} \int_{M} e^{f(a x)} \varphi(a x)[h(a x)]^{n} d \mu(a),
$$

which is equivalent to $\Theta(f+h)(\varphi)(x)-\Theta(f)(\varphi)(x)=\sum_{n=1}^{\infty} \frac{1}{n !} \Theta(f)\left(\varphi \cdot h^{n}\right)(x)$. This equality can be rewritten, by omitting the dependence on $x$ and $\varphi$, simply as follows

$$
\Theta(f+h)-\Theta(f)=\sum_{n=1}^{\infty} \frac{1}{n !} \Theta(f)\left((\cdot) h^{n}\right) .
$$

To justify the applicability of the Fubini Theorem in this case is sufficient to prove that the above sum converges in $(\mathcal{K},\|\cdot\|)$. We first observe that for any $h_{1}, \ldots, h_{k}$ in $\mathcal{K}$, the mapping $\varphi \mapsto$ $\Theta(f)\left(\varphi h_{1} \ldots h_{k}\right)$ from $\mathcal{K}$ to itself defines a continuous linear operator, i.e., $\Theta(f)\left((\cdot) h_{1} \ldots h_{k}\right) \in V$ whose its norm is bounded by

$$
\left\|\Theta(f)\left((\cdot) h_{1} \ldots h_{k}\right)\right\|_{V} \leq\left\|\mathscr{L}_{f}\right\|_{V}\left\|h_{1}\right\| \ldots\left\|h_{k}\right\| .
$$

which is proved by a routine computation using that $\mathscr{L}_{f} \in V$ and $(\mathcal{K},\|\cdot\|)$ is a Banach algebra. As a consequence of this inequality we get

$$
\sum_{n=1}^{\infty} \frac{1}{n !}\left\|\Theta(f)\left((\cdot)[h]^{n}\right)\right\|_{V} \leq \sum_{n=1}^{\infty} \frac{1}{n !}\left\|\mathscr{L}_{f}\right\|_{V}(\|h\|)^{n}=\left\|\mathscr{L}_{f}\right\|_{V}\left\{e^{\|h\|}-1\right\}
$$

which immediately implies that the series $\sum_{n=1}^{\infty}(1 / n !) \Theta(f)\left((\cdot)[h]^{n}\right)$ converges in $V$.

Claim 1. For any $k \in \mathbb{N}$ and $h_{1}, \ldots, h_{k} \in \mathcal{K}$, we have that

$$
D^{k} \Theta(f)\left(h_{1}, \ldots, h_{k}\right)=\Theta(f)\left((\cdot) h_{1}, \ldots, h_{k}\right) .
$$


The verification will be carried out by induction on $k$. In what follows $L^{k}=L^{k}(\mathcal{K}, V)$ denotes the set of all continuous $k$-linear functions $l: \mathcal{K} \times \ldots \times \mathcal{K} \rightarrow V$, from $\mathcal{K} \times \ldots \times \mathcal{K}(k-$ copies of $\mathcal{K}$ ) into $V$. The norm $\|\cdot\|_{L^{k}}$ of $L^{k}$ is given by

$$
\|l\|_{L^{k}}=\sup _{\substack{\left\|h_{i}\right\|=1 \\ i=1, \ldots, k}}\left\|l\left(h_{1}, \ldots, h_{k}\right)\right\|_{V}, \quad l \in L^{k} .
$$

Let us prove that the statement is true for $k=1$ : in fact, by using (11) we have,

$$
\Theta\left(f+h_{1}\right)-\Theta(f)=\Theta(f)\left((\cdot) h_{1}\right)+\mathcal{O}_{1}\left(h_{1}\right)
$$

where, $\mathcal{O}_{1}\left(h_{1}\right)=\sum_{n=2}^{\infty}(1 / n !) \Theta(f)\left((\cdot)\left[h_{1}\right]^{n}\right)$. The inequality (2) implies that $\left\|\Theta(f)\left((\cdot) h_{1}\right)\right\|_{V} \leq$ $\left\|\mathscr{L}_{f}\right\|_{V}\left\|h_{1}\right\|$ and thus the mapping $h_{1} \mapsto \Theta(f)\left((\cdot) h_{1}\right)$ is in $L^{1}$. Again, in view of the inequality (2) we have

$$
\left\|\mathcal{O}_{1}\left(h_{1}\right)\right\|_{V}=\left\|\sum_{n=2}^{\infty} \frac{1}{n !} \Theta(f)\left((\cdot)\left[h_{1}\right]^{n}\right)\right\|_{V} \leq \sum_{n=2}^{\infty} \frac{1}{n !}\left\|\mathscr{L}_{f}\right\|_{V}(\|h\|)^{n}
$$

showing that $\left(1 /\left\|h_{1}\right\|\right)\left\|\mathcal{O}_{1}\left(h_{1}\right)\right\|_{V} \rightarrow 0$, when $\left\|h_{1}\right\| \rightarrow 0$. Therefore, $D^{1} \Theta(f)\left(h_{1}\right)=\Theta(f)\left((\cdot) h_{1}\right)$ and the statement is true for $k=1$. Now, let us suppose the statement is true for $k-1, k \geq 2$, i.e.,

$$
D^{k-1} \Theta(f)\left(h_{1}, \ldots, h_{k-1}\right)=\Theta(f)\left((\cdot) h_{1} \ldots h_{k-1}\right), h_{1}, \ldots, h_{k-1} \in \mathcal{K} .
$$

We shall verify that the statement is true for $k$, i.e.

$$
D^{k} \Theta(f)\left(h_{1}, \ldots, h_{k}\right)=\Theta(f)\left((\cdot) h_{1} \ldots . h_{k}\right), h_{1}, \ldots, h_{k} \in \mathcal{K} .
$$

By the induction hypothesis (3), given $h_{1}, \ldots h_{k-1}, h_{k}$ and $h$ in $\mathcal{K}$ we have,

$$
\begin{aligned}
D^{k-1} \Theta\left(f+h_{k}\right)\left(h_{1}, \ldots, h_{k-1}\right)(h)-D^{k-1} \Theta(f) & \left(h_{1}, \ldots, h_{k-1}\right)(h) \\
& =\Theta\left(f+h_{k}\right)\left(h h_{1} \ldots h_{k-1}\right)-\Theta(f)\left(h h_{1} \ldots h_{k-1}\right) .
\end{aligned}
$$

From (11) follows that

$$
\begin{aligned}
D^{k-1} \Theta\left(f+h_{k}\right)\left(h_{1}, \ldots, h_{k-1}\right)(h)-D^{k-1} \Theta(f)\left(h_{1}, \ldots, h_{k-1}\right) & (h) \\
= & =\sum_{n=1}^{\infty} \frac{1}{n !} \Theta(f)\left(h h_{1} \ldots h_{k-1}\left[h_{k}\right]^{n}\right) .
\end{aligned}
$$

Clearly, the above equation shows that

$$
\begin{aligned}
D^{k-1} \Theta\left(f+h_{k}\right)\left(h_{1}, \ldots, h_{k-1}\right)-D^{k-1} \Theta(f)\left(h_{1}, \ldots, h_{k-1}\right) & \\
& \left.=\Theta(f)(\cdot) h_{1} \ldots h_{k-1} h_{k}\right)+\mathcal{O}_{k}\left(h_{k}\right)\left(h_{1} \ldots h_{k-1}\right),
\end{aligned}
$$

where $\mathcal{O}_{k}\left(h_{k}\right)$ is the element of $L^{k-1}$ given by

$$
\mathcal{O}_{k}\left(h_{k}\right)\left(h_{1} \ldots h_{k-1}\right)=\sum_{n=2}^{\infty} \frac{1}{n !} \Theta(f)\left((\cdot) h_{1} \ldots h_{k-1}\left[h_{k}\right]^{n}\right) .
$$


These upper bounds together with the inequality (2) enable us to conclude that the map $\left(h_{1} \ldots h_{k}\right) \mapsto \Theta(f)\left((\cdot) h_{1} \ldots h_{k}\right)$ is an element of $L^{k}$. The inequality (2) and the definition of $\mathcal{O}_{k}\left(h_{k}\right)$ give us the upper bound

$$
\left\|\mathcal{O}_{k}\left(h_{k}\right)\left(h_{1} \ldots h_{k-1}\right)\right\|_{V} \leq \sum_{n=2}^{\infty} \frac{1}{n !}\left\|\mathscr{L}_{f}\right\|_{V}\left\|h_{1}\right\| \ldots\left\|h_{k-1}\right\|\left(\left\|h_{k}\right\|\right)^{n}
$$

and consequently $\left(1 /\left\|h_{k}\right\|\right)\left\|\mathcal{O}_{k}\left(h_{k}\right)\right\|_{V} \rightarrow 0$, when $\left\|h_{k}\right\| \rightarrow 0$. Therefore, $D^{k} \Theta(f)\left(h_{1}, \ldots h_{k}\right)=$ $\Theta(f)\left((\cdot) h_{1} \ldots h_{k}\right)$ and the claim is proved.

By using the Claim 1 and the above estimates for the remaining the analyticity of the mapping $\mathcal{K} \ni f \mapsto \mathscr{L}_{f} \in V$ follows.

Analitycity of $\Theta^{*}$. Let $f, g$ and $h$ be potentials in $\mathcal{K}$ and $\phi^{*} \in \mathcal{K}^{*}$. From the expansion (1) for $\Theta(f+h)$ we get

$$
\begin{aligned}
\Theta^{*}(f+h)\left(\phi^{*}\right) g=\phi^{*}(\Theta(f+h)(g)) & =\phi^{*}\left(\sum_{n=0}^{\infty} \frac{1}{n !} \Theta(f)\left(g[h]^{n}\right)(\cdot)\right) \\
& =\sum_{n=0}^{\infty} \frac{1}{n !} \phi^{*}\left(\Theta(f)\left(g[h]^{n}\right)(\cdot)\right) \\
& =\sum_{n=0}^{\infty} \frac{1}{n !}\left(\Theta^{*}(f) \phi^{*}\right)\left(g[h]^{n}\right)(\cdot) .
\end{aligned}
$$

Claim 2. Consider the derivative map $D \Theta^{*}: \mathcal{K} \rightarrow L\left(\mathcal{K}, L\left(\mathcal{K}^{*}, \mathcal{K}^{*}\right)\right)$, then for any $f \in \mathcal{K}$ and $h \in \mathcal{K}$ we have that $D \Theta^{*}(f)(h): \mathcal{K}^{*} \rightarrow \mathcal{K}^{*}$ is given by $\left(D \Theta^{*}(f)(h)\left(\phi^{*}\right)\right) g=\left(\Theta^{*}(f)\left(\phi^{*}\right)\right)(g h)$. Indeed, consider $\mathcal{O}: \mathcal{K} \rightarrow L\left(\mathcal{K}^{*}, \mathcal{K}^{*}\right)$ defined by $\mathcal{O}(h)\left(\phi^{*}\right)=\sum_{n=2}^{\infty}(1 / n !) \Theta^{*}\left(\phi^{*}\right)\left((\cdot)[h]^{n}\right)$ then we have

$$
\begin{aligned}
\|\mathcal{O}(h)\|_{L\left(\mathcal{K}^{*}, \mathcal{K}^{*}\right)}=\sup _{\left\|\phi^{*}\right\|_{*}=1}\left\|\mathcal{O}(h)\left(\phi^{*}\right)\right\|_{*}=\sup _{\left\|\phi^{*}\right\|_{*}=1} \| & \sum_{n=2}^{\infty} \frac{1}{n !} \Theta^{*}(f)\left(\phi^{*}\right)\left((\cdot)[h]^{n}\right) \|_{*} \\
& \leq \sup _{\left\|\phi^{*}\right\|_{*}=1} \sum_{n=2}^{\infty} \frac{1}{n !} \underbrace{\left\|\Theta^{*}(f)\left(\phi^{*}\right)\left((\cdot)[h]^{n}\right)\right\|_{*}}_{I} .
\end{aligned}
$$

Next step is to upper bound the quantity $I$.

$$
\begin{aligned}
I=\left\|\Theta^{*}(f)\left(\phi^{*}\right)\left((\cdot)[h]^{n}\right)\right\|_{*} & =\sup _{\|g\|=1}\left\|\Theta^{*}(f)\left(\phi^{*}\right)\left((g)[h]^{n}\right)\right\|=\sup _{\|g\|=1}\left\|\phi^{*}\left(\Theta(f)\left((g)[h]^{n}\right)\right)\right\| \\
& \leq\left\|\phi^{*}\right\|_{*} \sup _{\|g\|=1}\left\|\Theta(f)\left((g)[h]^{n}\right)\right\| \leq\left\|\phi^{*}\right\|_{*}\|\Theta(f)\|_{V} \sup _{\|g\|=1}\left\|g[h]^{n}\right\| \\
& \leq\left\|\phi^{*}\right\|_{*}\|\Theta(f)\|_{V} \sup _{\|g\|=1}\|g\|\left\|[h]^{n}\right\| \leq \text { const. }\|h\|^{n} .
\end{aligned}
$$

By replacing (7) in (6) we get that

$$
\|\mathcal{O}(h)\|_{L\left(\mathcal{K}^{*}, \mathcal{K}^{*}\right)} \leq \sum_{n=2}^{\infty} \frac{1}{n !} \text { Const. }\|h\|
$$


therefore $\|\mathcal{O}(h)\|_{L\left(\mathcal{K}^{*}, \mathcal{K}^{*}\right)} /\|h\| \rightarrow 0$ when $\|h\| \rightarrow 0$.

It is possible to show that the higher orders derivatives $D^{k} \Theta^{*}: \mathcal{K} \rightarrow L\left(\mathcal{K}^{k}, L\left(\mathcal{K}^{*}, \mathcal{K}^{*}\right)\right)$ for $k \geq 2$, are given by the following expression

$$
D^{k} \Theta^{*}(f)\left(h_{1}, \ldots, h_{k}\right) \phi^{*}(g)=\Theta^{*}(f) \phi^{*}\left(h_{1} \cdots h_{k} g\right)=\phi^{*}\left(\mathscr{L}_{f}\left(h_{1} \cdots h_{k} g\right)\right) .
$$

The proof is similar to the previous one and so it will be omitted.

\section{The Ruelle Theorem on the Walters Space}

To simplify the notation for any $f \in C(\Omega)$ and $x, y \in \Omega$, we write

$$
S_{n} f(x) \equiv f(x)+f(\sigma(x))+\ldots+f\left(\sigma^{n-1}(x)\right) \quad \text { and } \quad d_{n}(x, y) \equiv \max _{0 \leq k<n} d_{\Omega}\left(\sigma^{k} x, \sigma^{k} y\right) .
$$

Definition 3.1. We say that a continuous function $f: \Omega \rightarrow \mathbb{R}$ is in the Walters class if given $\epsilon>0$ there exists $\eta>0$ such that

$$
\forall n \geq 1, \forall x, y \in \Omega, \quad d_{n}(x, y) \leq \eta \Longrightarrow\left|S_{n} f(x)-S_{n} f(y)\right| \leq \epsilon
$$

The space of all continuous function satisfying the above condition is denoted by $W(\Omega)$.

If a continuous function $f: \Omega \rightarrow \mathbb{R}$ satisfies the condition (7), we say that $f$ satisfies the Walters condition.

Definition 3.2. Consider a continuous function $f: \Omega \rightarrow \mathbb{R}$ and define $C_{f}(x, y)$ by

$$
C_{f}(x, y)=\sup _{n \geq 1} \sup _{\mathbf{a} \in M^{n}} S_{n} f(\mathbf{a} x)-S_{n} f(\mathbf{a} y) .
$$

We say that $f$ satisfies the weak Walters condition if $C_{f}(x, y) \rightarrow 0$ when $d(x, y) \rightarrow 0$.

Example. Consider the metric space $(M, d)$ were $M=[0,1]$ and $d=|\cdot|$. Now let $f$ be the potential defined on $\Omega=M^{\mathbb{N}}$ by $f(x)=x_{1}$, i.e., $f$ depends only on the first coordinate. We claim that $f$ satisfies the weak Walters condition but not the (strong) Walters condition. In fact, we have that $S_{n} f(\mathbf{a} x)=S_{n} f(\mathbf{a} y)=\sum_{i=1}^{n} a_{i}$ for any $\mathbf{a}=\left(a_{1}, \ldots, a_{n}\right) \in M^{n}$. Therefore $f$ clearly satisfies the weak Walters condition. Now we show that $f$ does not satisfy that Walters condition. Indeed, consider $x=(0,0 \ldots)$ the null vector and $y=(\eta, \eta, \ldots)$ for a small $\eta$. Notice that $d_{\Omega}(x, y)=d_{n}(x, y)=\eta$. On the other hand, $S_{n} f(x)=0$ and $S_{n} f(y)=n \cdot \eta$, then

$$
\left|S_{n} f(x)-S_{n} f(y)\right|=n \cdot \eta .
$$

From this is clear that $f$ does not satisfy the Walters condition.

On the other hand the opposite implication is always truth:

Proposition 3.3. Let be $f \in C(\Omega)$ satisfying the Walters condition then $f$ satisfies the weak Walters condition. 
Proof. Let $f \in W(\Omega)$, then by definition given $\epsilon>0$ arbitrarily there exists $\eta>0$ such that $\forall n \geq 1, \forall z, w \in \Omega$ with $d_{n}(z, w) \leq \eta$ we have $\left|S_{n} f(z)-S_{n} f(w)\right| \leq \epsilon$. Note that $d_{n}(\mathbf{a} x, \mathbf{a} y) \leq$ $d(x, y)$ for any $\mathbf{a} \in M^{n}$. Therefore $d(x, y) \leq \eta \Rightarrow d_{n}(\mathbf{a} x, \mathbf{a} y) \leq \eta \Rightarrow\left|S_{n} f(\mathbf{a} x)-S_{n} f(\mathbf{a} y)\right| \leq$ $\epsilon, \quad \forall \mathbf{a} \in M^{n}, \quad \forall n \geq 1$. By taking the supremum over all $\mathbf{a} \in M^{n}$ and $n \geq 1$ the result follows.

The space $W(\Omega)$ is clearly a linear space. Let $S$ denote the expansivity constant of the mapping $\sigma$. In [5] it was shown that for $s \in(0, S)$ the following expression

$$
\|f\|_{W_{s}}=2\|f\|_{0}+\sup _{n \geq 1} \max _{d_{n}(x, y) \leq s}\left|S_{n} f(x)-S_{n} f(y)\right|
$$

defines a family of equivalent norms and $\left(W(\Omega),\|\cdot\|_{W_{s}}\right)$ is a Banach Space. Since the family of norms $\left(\|\cdot\|_{W_{s}}\right)_{0<s<S}$ provides the same topology, there is no lost of generality in taking a particular value $s \in(0, S)$ and develop the theory with the norm $\|\cdot\|_{W} \equiv\|\cdot\|_{W_{s}}$.

En route to the proof of this work's main theorem, we need an extra structure of this space which is the structure of the Banach algebra. This is the content of the next lemma.

Lemma 3.4. The space $W(\Omega)$ with the norm $\|\cdot\|_{W}$ is a Banach algebra over $\mathbb{R}$. i.e., $W(\Omega)$ is a real Banach space and for all $f, g \in W(\Omega)$ we have $\|f g\|_{W} \leq\|f\|_{W}\|g\|_{W}$.

Proof. Let $f, g \in W(\Omega)$ and define $I \equiv I(f, g)$ by

$$
\begin{aligned}
I & \equiv \sup _{n \geq 1} \max _{d_{n}(x, y) \leq s}\left|S_{n}(f g)(x)-S_{n}(f g)(y)\right| \\
& =\sup _{n \geq 1} \max _{d_{n}(x, y) \leq s}\left|\sum_{j=1}^{n-1}(f g) \circ \sigma^{j}(x)-\sum_{j=1}^{n-1}(f g) \circ \sigma^{j}(y)\right| \\
& =\sup _{n \geq 1} \max _{d_{n}(x, y) \leq s} \mid \sum_{j=1}^{n-1}(f g) \circ \sigma^{j}(x)-\sum_{j=1}^{n-1} f \circ \sigma^{j}(x) g \circ \sigma^{j}(y) \\
& \quad+\sum_{j=1}^{n-1} f \circ \sigma^{j}(x) g \circ \sigma^{j}(y)-\sum_{j=1}^{n-1}(f g) \circ \sigma^{j}(y) \mid .
\end{aligned}
$$

By applying the Triangular Inequality we get that

$$
\begin{aligned}
& I \leq \sup _{n \geq 1} \max _{d_{n}(x, y) \leq s}\left|\sum_{j=1}^{n-1} f \circ \sigma^{j}(x)\left[g \circ \sigma^{j}(x)-g \circ \sigma^{j}(y)\right]\right| \\
& \quad+\sup _{n \geq 1} \max _{d_{n}(x, y) \leq s}\left|\sum_{j=1}^{n-1} g \circ \sigma^{j}(y)\left[f \circ \sigma^{j}(x)-f \circ \sigma^{j}(y)\right]\right| \\
& \leq\|f\|_{0} \cdot \sup _{n \geq 1} \max _{d_{n}(x, y) \leq s}\left|S_{n} g(x)-S_{n} g(y)\right|+\|g\|_{0} \cdot \sup _{n \geq 1} \max _{d_{n}(x, y) \leq s}\left|S_{n} f(x)-S_{n} f(y)\right| .
\end{aligned}
$$

The last upper bound readily implies that

$$
\|f g\|_{W} \leq 2\|f g\|_{0}+\|f\|_{0} \sup _{n \geq 1} \max _{d_{n}(x, y) \leq s}\left|S_{n} g(x)-S_{n} g(y)\right|+\|g\|_{0} \sup _{n \geq 1} \max _{d_{n}(x, y) \leq s}\left|S_{f}(x)-S_{n} f(y)\right| .
$$


On the other hand, it follows from the definition of $\|\cdot\|_{W}$ that

$$
\begin{aligned}
\|f\|_{W} \cdot\|g\|_{W}=4\|f\|_{0}\|g\|_{0} & +2\|f\|_{0} \cdot \sup _{n \geq 1} \max _{d_{n}(x, y) \leq s}\left|S_{n} g(x)-S_{n} g(y)\right| \\
& +2\|g\|_{0} \cdot \sup _{n \geq 1} \max _{d_{n}(x, y) \leq s}\left|S_{f}(x)-S_{n} f(y)\right| \\
& +\sup _{n \geq 1} \max _{d_{n}(x, y) \leq s}\left|S_{n} g(x)-S_{n} g(y)\right| \cdot \sup _{n \geq 1} \max _{d_{n}(x, y) \leq s}\left|S_{n} f(x)-S_{n} f(y)\right| .
\end{aligned}
$$

This identity and the previous estimates readily implies that $\|f g\|_{W} \leq\|f\|_{W} \cdot\|g\|_{W}$.

Proposition 3.5. If $f \in W(\Omega)$, then $\mathscr{L}_{f}(W(\Omega)) \subset W(\Omega)$.

Proof. We claim that for any fixed $a \in M$, if $f \in W(\Omega)$ then the function $x \mapsto f(a x)$ also belongs to $W(\Omega)$. In fact, given $\epsilon>0$ we choose $\eta>0$ such that the Walters condition (77) is satisfied for $f$. Note that $d_{n}(x, y) \leq \delta \Rightarrow d_{n}(a x, a y) \leq d_{n}(x, y) \leq \eta$. From the definition we have that $d_{n}(x, y) \leq \eta$ implies $\left|S_{n} f(a x)-S_{n} f(a y)\right| \leq \epsilon$ for all $n>0$ and therefore the claim is proved.

The next step is to prove that the function $r: \Omega \rightarrow \mathbb{R}$ given by $r(x)=\int_{M} h(a x) d \mu(a)$ belongs to $W(\Omega)$ whenever $h \in W(\Omega)$. From the previous claim follows that $x \mapsto h(a x)$ is in $W(\Omega)$ since $h \in W(\Omega)$. Given $\epsilon>0$ we now choose $\eta>0$ such that the condition (7) is satisfied for $x \mapsto h(a x)$. Since $d_{n}(x, y) \leq \eta \Rightarrow d_{n}(a x, a y) \leq \eta$, the Walters condition for $r$ follows from the inequality

$$
\left|S_{n} r(x)-S_{n} r(y)\right| \leq \int_{M}\left|S_{n} h(a x)-S_{n} h(a y)\right| d \mu(a) \leq \epsilon \cdot \mu(M)
$$

By hypothesis the potential $f \in W(\Omega)$, since the Walters space is a Banach algebra we have that $\exp (f) \in W(\Omega)$. For the same reason, for any $\varphi \in W(\Omega)$ we have $\varphi \cdot \exp (f) \in W(\Omega)$. As argued above, for any $a \in M$, the mapping $x \mapsto \varphi(a x) \cdot \exp (f(a x))$ belongs to $W(\Omega)$. Using the result proved above for the function $r$, with $h(x)=\varphi(x) \exp (f(x))$, it follows that the mapping

$$
x \mapsto \int_{M} \varphi(a x) \exp (f(a x)) d \mu(a) \equiv \mathscr{L}_{f}(\varphi)(x)
$$

is in the Walters space for any $\varphi \in W(\Omega)$ which finishes the proof.

\section{The Ruelle Theorem On Walters space}

The proof of this version of the Ruelle Theorem is inspired in the original proof presented in Walters [43].

Let be $f$ potential in $C(\Omega)$. Consider the function $C_{f}$ given by

$$
C_{f}(x, y)=\sup _{n \geq 1} \sup _{\mathbf{a} \in M^{n}} \sum_{i=0}^{n-1}\left(f\left(\sigma^{i}(\mathbf{a} x)\right)-f\left(\sigma^{i}(\mathbf{a} y)\right) .\right.
$$


We say that $f$ satisfies the weak Walters condition if $C_{f}(x, y) \rightarrow 0$, when $d_{\Omega}(x, y) \rightarrow 0$.

To prove the Ruelle Theorem we concentrate attention on a certain subclass of $C(\Omega)$ which is given by

$$
G_{0}(\Omega)=\left\{g \in C(\Omega): g>0 \text { and } \int_{M} g(a x) d \mu(a)=1 \forall x \in \Omega\right\} .
$$

If $f: \Omega \rightarrow \mathbb{R}$ is a potential given by $f=\log g$, where $g \in G_{0}(\Omega)$, then the weak Walters condition for $f$ can be rephrased in terms of $g$ by saying that

$$
D_{g}(x, y)=\sup _{n \geq 1} \sup _{\mathbf{a} \in M^{n}} \prod_{i=0}^{n-1} \frac{g\left(\sigma^{i} \mathbf{a} x\right)}{g\left(\sigma^{i} \mathbf{a} y\right)}
$$

exists, is bounded for a constant $D_{g}$ and $D_{g}(x, y) \rightarrow 1$ when $d_{\Omega}(x, y) \rightarrow 0$. Equivalently:

$$
D_{g}^{\star}(x, y)=\sup _{n \geq 1} \sup _{\mathbf{a} \in M^{n}}\left|\prod_{i=0}^{n-1} \frac{g\left(\sigma^{i} \mathbf{a} x\right)}{g\left(\sigma^{i} \mathbf{a} y\right)}-1\right| \leq D_{g}-1
$$

for all $x, y$ with $d_{\Omega}(x, y)<\epsilon_{0}$ and $D_{g}^{\star}(x, y) \rightarrow 0$ when $d_{\Omega}(x, y) \rightarrow 0$.

Theorem 4.1. Let $g \in G_{0}(\Omega)$ be a function such that $\log g$ satisfies the weak Walters condition. Then there is a probability measure $\nu: \mathscr{B}(\Omega) \rightarrow[0,1]$ such that

$$
\mathscr{L}_{\log g}^{n} \varphi \stackrel{\left\|^{\prime}\right\|_{0}}{\longrightarrow} \nu(\varphi)
$$

for all $\varphi \in C(\Omega)$. Moreover $\nu$ is the unique probability measure satisfying $\mathscr{L}_{\log g}^{*} \nu=\nu$.

Proof. The proof is based on a simple modification of the arguments given in [44] and it is presented here for the reader's convenience.

Let us introduce the temporary notation $\mathscr{L}$ for $\mathscr{L}_{\log g}$. We begin by proving that $\left\{\mathscr{L}^{n} \varphi, n \geq\right.$ $0\}$ is equicontinuous family for any fixed $\varphi$ satisfying the weak Walters condition. Indeed, from the definition of the Ruelle operator we have

$$
\begin{aligned}
\left|\mathscr{L}^{n} \varphi(x)-\mathscr{L}^{n} \varphi(y)\right| & \leq\left|\int_{M^{n}}\left[\exp \left(S_{n} \log g(\mathbf{a} x)\right) \varphi(\mathbf{a} x)-\exp \left(S_{n} \log g(\mathbf{a} y)\right) \varphi(\mathbf{a} y)\right] \prod_{i=1}^{n} d \mu\left(a_{i}\right)\right| \\
& \leq\left|\int_{M^{n}} \prod_{i=0}^{n-1} g\left(\sigma^{i}(\mathbf{a} x)\right)[\varphi(\mathbf{a} x)-\varphi(\mathbf{a} y)] \prod_{i=0}^{n} d \mu\left(a_{i}\right)\right| \\
& +\left|\int_{M^{n}} \varphi(\mathbf{a} y)\left[\prod_{i=0}^{n-1} g\left(\sigma^{i}(\mathbf{a} x)\right)-\prod_{i=0}^{n-1} g\left(\sigma^{i}(\mathbf{a} y)\right)\right] \prod_{i=0}^{n} d \mu\left(a_{i}\right)\right|
\end{aligned}
$$

The two terms in the rhs above can be bounded by

$$
\begin{aligned}
\sup _{\mathbf{a} \in M^{n}}\{|\varphi(\mathbf{a} x)-\varphi(\mathbf{a} y)|\} \mid \int_{M^{n}} & \prod_{i=0}^{n-1} g\left(\sigma^{i}(\mathbf{a} x)\right) \prod_{i=1}^{n} d \mu\left(a_{i}\right) \mid \\
& \quad+\|\varphi\|_{0} \int_{M^{n}} \prod_{i=0}^{n-1} g\left(\sigma^{i}(\mathbf{a} y)\right)\left|\frac{\prod_{i=0}^{n-1} g\left(\sigma^{i}(\mathbf{a} x)\right)}{\prod_{i=0}^{n-1} g\left(\sigma^{i}(\mathbf{a} y)\right)}-1\right| \prod_{i=1}^{n} d \mu\left(a_{i}\right) .
\end{aligned}
$$


Since $g \in G_{0}(\Omega)$ it follows from the Fubini Theorem that the iterated integral on the first term above is equal to one. The second term can be bound similarly by using the definition of $D_{g}^{\star}(x, y)$, which give us the following inequality

$$
\left|\mathscr{L}^{n} \varphi(x)-\mathscr{L}^{n} \varphi(y)\right| \leq \sup _{\mathbf{a} \in M^{n}}\{|\varphi(\mathbf{a} x)-\varphi(\mathbf{a} y)|\}+\|\varphi\|_{0} \cdot D_{g}^{\star}(x, y) .
$$

Since $\varphi$ is a continuous function and $\log g$ satisfies the weak Walters condition the above inequality ensures that the family $\left\{\mathscr{L}^{n} \varphi, n \geq 0\right\}$ is equicontinuous.

Recalling that $g \in G_{0}(\Omega)$ we get from the definition of the Ruelle operator for all $n \in \mathbb{N}$ that $\left\|\mathscr{L}^{n} \varphi\right\|_{0} \leq\|\varphi\|_{0}$ for any $\varphi \in C(\Omega)$. This inequality implies that the closure in the uniform topology of $\left\{\mathscr{L}^{n} \varphi, n \geq 0\right\}$ is uniformly bounded in $C(\Omega)$. Therefore we can apply the ArzelaAscoli Theorem for the family $\left\{\mathscr{L}^{n} \varphi, n \geq 0\right\}$ to guarantee that there exist a subsequence $\left(n_{i}\right) \subset \mathbb{N}$ and function $\varphi^{\star} \in C(\Omega)$ so that $\mathscr{L}^{n_{i}} \varphi \longrightarrow \varphi^{\star}$ uniformly.

Let us proceed by showing that $\varphi^{\star}$ is a constant function. Notice that the identity $\mathscr{L}(1)=1$ implies the following inequalities $\min (\varphi) \leq \min (\mathscr{L}(\varphi)) \leq \cdots \leq \min \left(\varphi^{\star}\right)$. Claim 1. For any $k \in$ $\mathbb{N}$ we have $\min \left(\mathscr{L}^{k} \varphi^{\star}\right)=\min \left(\varphi^{\star}\right)$. Indeed, we have that $\min \left(\mathscr{L}^{k} \varphi^{\star}\right)=\min \left(\mathscr{L}^{k}\left(\lim \mathscr{L}^{n_{i}} \varphi\right)\right)=$ $\min \left(\lim \mathscr{L}^{n_{i}+k} \varphi\right)=\lim \left(\min \left(\mathscr{L}^{n_{i}+k} \varphi\right)\right)=\min \left(\varphi^{\star}\right)$, where the last equality it follows from the monotonicity of the sequence $\min \mathscr{L}^{k} \varphi$ and $\min \mathscr{L}^{n_{i}} \varphi \longrightarrow \min \varphi^{\star}$. Given $\epsilon>0$ choose $x \in \Omega$ and $N \in \mathbb{N}$ such that $\min \left(\mathscr{L}^{N} \varphi^{\star}\right)=\mathscr{L}^{N} \varphi^{\star}(x)$ and $\left\{\mathbf{a} x, \mathbf{a} \in M^{N}\right\}$ is $\epsilon$-dense in $\Omega$. Claim 2. For all $y \in \sigma^{-N} x$ we have $\varphi^{\star}(y)=\min \left(\varphi^{\star}\right)$. From the Claim 1 and the choice of $x$, we have $\mathscr{L}^{N} \varphi^{\star}(x)=\min \left(\varphi^{\star}\right)$. Let $z \in \Omega$ be such that $\varphi^{\star}(z)=\min \left(\varphi^{\star}\right)$, then

$$
\int_{M^{N}} g(\mathbf{a} x) g(\sigma \mathbf{a} x) \cdots g\left(\sigma^{N-1} \mathbf{a} x\right) \varphi^{\star}(\mathbf{a} x) \prod_{i=1}^{N} d \mu\left(a_{i}\right)=\mathscr{L}^{N} \varphi^{\star}(x)=\min \left(\varphi^{\star}\right)=\varphi^{\star}(z) .
$$

By using the identity $1=\mathscr{L}_{\log g}^{N} 1(x)$, it follows from the above equation that

$$
0=\int_{M^{N}} g(\mathbf{a} x) g(\sigma \mathbf{a} x) \cdots g\left(\sigma^{N-1} \mathbf{a} x\right)\left[\varphi^{\star}(\mathbf{a} x)-\varphi^{\star}(z)\right] \prod_{i=1}^{N} d \mu\left(a_{i}\right)
$$

By using the continuity of $\varphi^{\star}$ and the assumption $\operatorname{supp}(\mu)=M$ it is easy to see that $\varphi^{\star}(\mathbf{a} x)=$ $\varphi^{\star}(z)$ for any $\mathbf{a} \in M^{N}$. Since $\varphi^{\star}$ is continuous and constant over $\left\{\mathbf{a} x, \mathbf{a} \in M^{N}\right\}$, which is $\epsilon$-dense in $\Omega$, follows that $\varphi^{\star}$ is a constant function.

We now shown the existence and uniqueness of the fixed point for $\mathscr{L}^{*} \equiv \mathscr{L}_{\log g}^{*}$. Define the linear functional $F: C(\Omega) \rightarrow \mathbb{R}$ by $F(\varphi)=\varphi^{\star}$. The functional $F$ sends the cone of positive continuous functions to itself and satisfies $F(1)=1$. Then it follows from the Riesz-Markov Theorem that there exists an unique Borel probability measure $\nu \in \mathcal{M}(\Omega)$ that represents $F$. It is a simple matter to show that $\mathscr{L}^{*} \nu=\nu$. For the uniqueness suppose that there exists another probability measure $\gamma \in \mathcal{M}(\Omega)$ such that $\mathscr{L}^{*} \gamma=\gamma$. Of course, $\left(\mathscr{L}^{*}\right)^{n} \gamma=\gamma$ for every $n \in \mathbb{N}$ and

$$
\int_{\Omega} \varphi d \gamma=\int_{\Omega} \varphi d\left[\left(\mathscr{L}^{*}\right)^{n} \gamma\right]=\int_{\Omega} \mathscr{L}^{n} \varphi d \gamma=\int_{\Omega} \lim _{n \rightarrow \infty} \mathscr{L}^{n} \varphi d \gamma=\int_{\Omega} \varphi^{\star} d \gamma=\varphi^{\star}=\int_{\Omega} \varphi d \nu .
$$

Since $\varphi \in C(\Omega)$ is arbitrary it follows that $\gamma=\nu$. 
Lemma 4.2. Let $f$ satisfying the weak Walters condition, then $\forall \epsilon>0$, there exists $N>0$ and $a \in \mathbb{R}$ such that $\forall x, y \in \Omega, \quad \exists w \in \sigma^{-N} x \cap B_{d_{\Omega}}(y, \epsilon)$ with $S_{N} f(w) \geq a$.

Proof. See [44] page 126.

Lemma 4.3. Let $f \in C(\Omega)$ be a potential. Then there exists a real number $\lambda>0$ and a Borel probability measure $\nu \in \mathcal{M}(\Omega)$ such that $\mathscr{L}_{f}^{*} \nu=\lambda \nu$.

Proof. The mapping $\gamma \mapsto \mathscr{L}_{f}^{*} \gamma /\left(\mathscr{L}_{f}^{*} \gamma\right)(1)$ define a continuous function from $\mathcal{M}(\Omega)$ to itself. The Schauder-Tychonoff fixed point theorem ensures the existence of a fixed point $\nu$ for this mapping. By taking $\lambda=\left(\mathscr{L}_{f}^{*} \nu\right)(1)$ the theorem follows.

We are now able to prove the main theorem of this section which is the Ruelle Theorem for Walters potentials defined over an infinite cartesian product of general metric compact spaces.

Theorem 4.4. Let $f$ be a potential satisfying the weak Walters condition and consider the Ruelle operator $\mathscr{L}_{f}: C(\Omega) \rightarrow C(\Omega)$ associated to $f$. Then there are a real number $\lambda_{f}>0, a$ strictly positive continuous function $h_{f}$ and a unique Borel probability measure $\nu_{f}$ such that

i) $\mathscr{L}_{f} h_{f}=\lambda_{f} h_{f}, \mathscr{L}_{f}^{*} \nu_{f}=\lambda_{f} \nu_{f}$.

ii) For any $\varphi \in C(\Omega)$ we have

$$
\left\|\lambda_{f}^{-n} \mathscr{L}_{f}^{n} \varphi-h_{f} \int_{\Omega} \varphi d \nu_{f}\right\|_{0} \longrightarrow 0, \quad \text { when } n \rightarrow \infty .
$$

Proof. The proof will be divided into three steps.

Claim 1. Let $\nu$ and $\lambda$ as given by the Lemma 4.3. Then for any $f$ satisfying the weak Walters condition and $\epsilon_{0}>0$ the set

$$
\Lambda=\left\{\varphi \in C(\Omega): \varphi \geq 0, \nu(\varphi)=1 \text { and } \varphi(x) \leq \exp \left(C_{f}(x, y)\right) \varphi(y) \text { if } d_{\Omega}(x, y)<\epsilon_{0}\right\}
$$

is convex, closed, bounded and uniformly equicontinuous.

Let us first prove that $\Lambda$ is not empty. Indeed, for any $x, y \in \Omega$ we have

$$
\begin{aligned}
\mathscr{L}_{f} 1(x)=\int_{M} e^{f(a x)} d \mu(a) & =\int_{M} e^{f(a y)} e^{f(a x)-f(a y)} d \mu(a) \\
\leq & \exp \left(\sup _{a \in M} f(a x)-f(a y)\right) \int_{M} e^{f(a y)} d \mu(a) \leq \exp \left(C_{f}(x, y)\right) \mathscr{L}_{f} 1(y) .
\end{aligned}
$$

The set $\Lambda$ is clearly closed and convex. Now we shall prove that $\Lambda$ is a bounded set. Let $x, y \in \Omega$. By the Lemma 4.2 given $\epsilon>0$ and $a \in \mathbb{R}$ there is $N \in \mathbb{N}$ and $y_{0}=\mathbf{a}_{0} x$, where $\mathbf{a}_{0}=a_{1} \ldots a_{N}$, such that $d_{\Omega}\left(y_{0}, y\right)<\epsilon$ and $S_{N} f\left(y_{0}\right) \geq a$. Given $\delta_{1}>0$ it follows from the continuity of $\varphi$ that we can choose $\delta>0$ such that for any point $\mathbf{a}$ in the closed ball $B\left[\mathbf{a}_{0}, \delta\right] \subset M^{N}$ we have 
$S_{N} f(\mathbf{a} x) \geq a-\delta_{1}$. In particular, we can choose $\delta$ such that $B\left[y_{0}, \delta\right] \subset B[y, \epsilon]$. Therefore it follows from the definition of the Ruelle operator and the choice of $\delta$ that

$$
\begin{aligned}
\mathscr{L}^{N} \varphi(x) & =\int_{M^{N}} e^{S_{N} f(\mathbf{a} x)} \varphi(\mathbf{a} x) \prod_{i=1}^{N} d \mu\left(a_{i}\right) \\
& =\int_{M^{N} \backslash B\left[\mathbf{a}_{0}, \delta\right]} e^{S_{N} f(\mathbf{a} x)} \varphi(\mathbf{a} x) \prod_{i=1}^{N} d \mu\left(a_{i}\right)+\int_{B\left[\mathbf{a}_{0}, \delta\right]} e^{S_{N} f(\mathbf{a} x)} \varphi(\mathbf{a} x) \prod_{i=1}^{N} d \mu\left(a_{i}\right) \\
& \geq \int_{B\left[\mathbf{a}_{0}, \delta\right]} e^{S_{N} f(\mathbf{a} x)} \varphi(\mathbf{a} x) \prod_{i=1}^{N} d \mu\left(a_{i}\right) \\
& \geq \mu \times \ldots \times \mu\left(B\left[\mathbf{a}_{0}, \delta\right]\right) e^{a-\delta_{1}} \varphi\left(w_{0}\right),
\end{aligned}
$$

where $w_{0}$ minimizes the function $\left(a_{1}, \ldots, a_{N}\right) \mapsto \varphi\left(a_{1} \ldots a_{N} x\right)$ in $B[y, \delta]$. Now observe that $w_{0} \in$ $B[y, \epsilon]$, by using the compactness of $\Omega$ and the definition of $\Lambda$ we get for all $x, y \in \Omega$ the following inequality $\varphi(y) \leq$ Const $\cdot e^{\delta_{1}-a} \mathscr{L}^{N} \varphi(x)$. Recalling that $\mathscr{L}_{f}^{*} \nu=\lambda \nu$ and $\nu(\varphi)=1$, we obtain by integrating both sides of the previous inequality that $\varphi(y) \leq$ Const $\cdot e^{C+\delta_{1}-a} \nu\left(\mathscr{L}^{N} \varphi(x)\right)=$ Const $\cdot e^{C+\delta_{1}-a} \lambda^{N}$. Hence $\Lambda$ is bounded. The uniform equicontinuity of $\Lambda$ is proved as in [44, p. 129] mutatis mutandis.

Claim 2. The operator $\lambda^{-1} \mathscr{L}_{f}$ maps $\Lambda$ into $\Lambda$.

Let $\varphi \in \Lambda$ and $x, y \in \Omega$ with $d_{\Omega}(x, y)<\epsilon_{0}$. Then

$$
\begin{aligned}
\frac{1}{\lambda} \mathscr{L} \varphi(x) & =\frac{1}{\lambda} \int_{M} e^{f(a x)} \varphi(a x) d \mu(a) \\
& \leq \frac{1}{\lambda} \int_{M} e^{f(a y)} \varphi(a y) \underbrace{\left(e^{f(a x)-f(a y)} e^{C_{f}(a x, a y)}\right)}_{\leq e^{C_{f}(x, y)}} d \mu(a) \\
& \leq \frac{1}{\lambda} \int_{M} e^{f(a y)} \varphi(a y) e^{C_{f}(x, y)} d \mu(a) \\
& =\frac{1}{\lambda} \mathscr{L}_{f} \varphi(y) e^{C_{f}(x, y)}
\end{aligned}
$$

where the inequality $e^{f(a x)-f(a y)} e^{C_{f}(a x, a y)} \leq e^{C_{f}(x, y)}$ is justified by observing that $C_{f}(a x, a y)$ is equal to

$$
\sup _{n \geq 1} \sup _{\mathbf{a} \in M^{n}}\left\{(f(\mathbf{a} a x)-f(\mathbf{a} a y))+(f(\sigma \mathbf{a} x)-f(\sigma \mathbf{a} a y))+\cdots+\left(f\left(\sigma^{n-1} \mathbf{a} a x\right)-f\left(\sigma^{n-1} \mathbf{a} a y\right)\right)\right\} .
$$

From this is clearly that $C_{f}(a x, a y)+(f(a x)-f(a y)) \leq C_{f}(x, y)$.

Claim 3. If $g=e^{f} h /(\lambda h \circ T)$, then $g \in G_{0}(\Omega)$ and $D_{g}^{\star}(x, y) \rightarrow 0$ when $d_{\Omega}(x, y) \rightarrow 0$. The proof is similar to the one given by [44, p. 130].

The Claims 1 and 2 allow us to use the Schauder-Tychonoff fixed point theorem to obtain a fixed point $h \in \Lambda$ for the operator $\lambda^{-1} \mathscr{L}$. This fixed point $h$ satisfies $\mathscr{L} h=\lambda h, \nu(h)=1$ 
and $h(x) \leq e^{C_{f}(x, y)} h(y)$ whenever $d_{\Omega}(x, y)<\epsilon_{0}$. We shall show that $h>0$. Suppose that there exist some $x \in \Omega$ such that $h(x)=0$. For all $n \in \mathbb{N}$ we have $\mathscr{L}^{n} h(x)=\lambda^{n} h(x)=0$, so $h$ must be 0 on the set $\left\{\sigma^{-n} x, n \in \mathbb{N}\right\}$, which is dense, using that $\mu$ has full support we have $h \equiv 0$ contradicting the fact that $\nu(h)=1$.

By the Theorem 4.1] we have that $\mathscr{L}_{\log g}^{n} \varphi \stackrel{|\cdot|_{0}}{\longrightarrow} \mu(\varphi)$ for all $\varphi \in C^{0}(\Omega)$, where $\mu \in \mathcal{M}(\Omega)$ is the fixed point of $\mathscr{L}_{\log g}^{*}$ in $\mathcal{M}(\Omega)$. On the other hand

$$
\frac{1}{\lambda^{n}} \mathscr{L}_{f}^{n} \varphi(x)=h(x)\left(\mathscr{L}_{\log g}^{n}(\varphi / h)(x)\right)
$$

then it follows that $1 / \lambda^{n} \mathscr{L}_{f}^{n} \varphi \stackrel{|\cdot|_{0}}{\longrightarrow} h \cdot \mu(\varphi / h)$. We shall show that $\mu(\varphi / h)=\nu(\varphi)$. Let be $m \in \mathcal{M}(\Omega)$ defined by $m(\varphi)=\nu(h \varphi)$. Then

$$
m\left(\mathscr{L}_{\log g} \varphi\right)=\nu\left(h \cdot \mathscr{L}_{\log g} \varphi\right)=\frac{1}{\lambda}=\nu\left(\mathscr{L}_{f}(\varphi \cdot h)=m(\varphi) .\right.
$$

\section{Spectral Gap and Analyticity of the Pressure}

We mean by "presence of the spectral gap" in the Ruelle operator the existence of a single isolated eigenvalue of maximum modulus. The presence of the spectral gap in the Ruelle operator is the key property to prove analyticity of the pressure and also implies the exponential decay of correlations with respect to the Gibbs measures. These are classical results and very well known for Hölder potentials when the state space $M$ is finite. We shall here analyze the generalizations of this results in the sense of the state space $M$ and the regularity of the potential.

The main difference between the Ruelle Theorem operator for $f \in W(\Omega)$ and for $f \in C^{\gamma}(\Omega)$ is the fact that in the first case we do not have much information about the spectrum of $\mathscr{L}_{f}$. In particular, it seems a hard problem to decide whether we have presence or absence of the spectral gap for the Ruelle operator $\mathscr{L}_{f}$, which is crucial property to get deep understanding of the associated Gibbs measure.

Theorem 5.1. Let $\mathcal{K} \subset C(\Omega)$ be an invariant subspace of $C(\Omega)$. A sufficient condition for the analyticity of the pressure is the analyticity of the map $\mathcal{K} \ni f \mapsto \mathscr{L}_{f} \in \mathcal{K}$ and the presence of the spectral gap in the spectrum of the Ruelle operator.

In order to prove the Theorem 5.1 we shall use the following lemma, which seems to be a well known fact on the community. We decided to give a proof for this lemma either to keep the text as self contained as possible and because we were not able to find its reference.

In this paper we use the expression simple eigenvalue to refer to an eigenvalue $\lambda$ of an operator $T: X \rightarrow X$ such that the image of the spectral projector $\pi_{L}=\int_{\partial D}(\lambda I-L)^{-1} d \lambda$ is an uni-dimensional subspace of $X$. 
Lemma 5.2. Let $T: X \rightarrow X$ be a bounded linear operator possessing an isolated simple eigenvalue $\lambda \in \mathbb{C}$. Let $D$ be a closed disc centered at $\lambda$ such that $D \cap \operatorname{spec}(T)=\{\lambda\}$. Then there is a neighborhood $U$ of $T$ in $L(X, X)$ so that the mapping $U \ni L \mapsto \lambda(L) \in \mathbb{C}$, where $\lambda(L)$ is the unique point in $\operatorname{spec}(L) \cap \operatorname{int}(D)$ is well defined and moreover this mapping is analytic.

Corollary 5.3. For any fixed $0<\gamma \leq 1$ both mappings

$$
C^{\gamma}(\Omega) \ni f \mapsto h_{f} \in C^{\gamma}(\Omega) \quad \text { and } \quad C^{\gamma}(\Omega) \ni f \mapsto \nu_{f} \in\left(C^{\gamma}(\Omega)\right)^{*}
$$

are analytic.

Proof. Choose $f$ arbitrarily in $C^{\gamma}(\Omega)$. Let $U$ and $\tilde{U}$ be neighborhoods of $\mathscr{L}_{f}$ and $\mathscr{L}_{f}^{*}$, respectively such that for all $\mathscr{L}_{g} \in U$ and $\mathscr{L}_{g}^{*} \in \tilde{U}$ the images of the spectral projectors $\pi_{\mathscr{L}_{g}}$ and $\pi_{\mathscr{L}_{g}^{*}}$ are the one-dimensional spaces associated to the eigenvalue $\lambda_{g}$. Consider a neighborhood $W \subset C^{\gamma}(\Omega)$ of $f$ so that $\mathscr{L}_{g} \in U$ and $\mathscr{L}_{g}^{*} \in \tilde{U}$, respectively whenever $g \in W$. Therefore we have that $\pi_{\mathscr{L}_{g}^{*}} \nu_{f}=c \cdot \nu_{g}$ then $c=c \cdot \int_{\Omega} 1 d \nu_{g} \equiv c \cdot\left\langle\nu_{g}, 1\right\rangle=\left\langle\pi_{\mathscr{L}_{g}^{*}} \nu_{f}, 1\right\rangle$ so $\nu_{g}=\left\langle\pi_{\mathscr{L}_{g}^{*}} \nu_{f}, 1\right\rangle^{-1} \cdot \pi_{\mathscr{L}_{g}^{*}} \nu_{f}$ Being the rhs of the last expression a composition of analytic functions follows that $g \mapsto \nu_{g}$ is analytic in a neighborhood of $f$. On the other hand, $\pi_{\mathscr{L}_{q}} h_{f}=C \cdot h_{g}$ so by a suitable choice of the eigenfunction it follows that $C=C \cdot\left\langle\nu_{g}, h_{g}\right\rangle=\left\langle\nu_{g}, \pi_{\mathscr{L}_{g}} h_{f}\right\rangle$, therefore $h_{g}=\left\langle\nu_{g}, \pi_{\mathscr{L}_{g}} h_{f}\right\rangle^{-1} \cdot \pi_{\mathscr{L}_{g}} h_{f}$ which is again a composition of analytic functions and so $g \mapsto h_{g}$ is analytic in a neighborhood of $f$.

Now we are ready to state and prove the main result of this section.

Theorem 5.4. The function defined by $C^{\gamma}(\Omega) \ni f \rightarrow P(f) \in \mathbb{R}$ is real analytic function.

Proof. The proof is based on the analyticity of both functions $C^{\gamma}(\Omega) \ni f \mapsto \mathscr{L}_{f}$ and $U \ni T \mapsto$ $\lambda(T) \in \mathbb{R}$. The analyticity of the first map is the content of the Lemma (2.4), whose detailed proof is given in [39] Theorem 3.5. The analyticity of the second mapping it follows from the Theorem 2.1, which assures that $\mathscr{L}_{f}: C^{\gamma}(\Omega) \rightarrow C^{\gamma}(\Omega)$ has the spectral gap property and the Lemma 5.2. To finish the proof it is enough to observe that the mapping $C^{\gamma}(\Omega) \ni f \rightarrow P(f) \in \mathbb{R}$ is given by $P(f)=\log \lambda_{f}$. Indeed, the rhs is the composition of the following analytic mappings: $\log , C^{\gamma}(\Omega) \ni f \mapsto \mathscr{L}_{f}$ and $U \ni T \mapsto \lambda(T) \in \mathbb{R}$.

Proof of the Lemma 5.2. The argument is based on the following two claims.

Claim 1. There is a neighborhood $U$ of $T$ in $L(X, X)$ such that $\operatorname{spec}(L) \cap \partial D=\varnothing$ for all $L \in U$. This claim is proved by contradiction. Suppose that for each $n$ there exist $L_{n} \in B(T, 1 / n) \subset$ $L(X, X)$ so that $\lambda_{L_{n}} \in \operatorname{spec}\left(L_{n}\right) \cap \partial D$ and the operator $\lambda_{L_{n}} I-L_{n}$ is not invertible. Since $\partial D$ is compact we can find a convergent subsequence $\left\{\lambda_{L_{n_{j}}}\right\} \subset \partial D$ so that $\lambda_{L_{n_{j}}} \rightarrow \lambda_{L} \in \partial D$. Since $L_{n} \rightarrow T$ we have that $\left(\lambda_{L_{n_{j}}} I-L_{n_{j}}\right) \rightarrow\left(\lambda_{L} I-T\right)$ in the strong topology. Since $\left(\lambda_{L} I-T\right)$ is invertible and the space of the invertible bounded linear operators is open we thus have a contradiction.

Claim 2. We can shrink $U$ so that for all $L \in U$ we have that $\operatorname{spec}(L) \cap \operatorname{int}(D)$ is a simple eigenvalue of $L$. In fact, for each $L \in U$ let $\pi_{L}$ be the spectral projector given by

$$
\pi_{L}=\int_{\partial D}(\lambda I-L)^{-1} d \lambda .
$$


Notice that the mapping $U \ni L \mapsto \pi_{L} \in L(X, X)$ is continuous, so by the Proposition (8.7) if necessary one can shrink $U$ so that for all $L \in U$ the application $\pi_{L}$ has the same rank as $\pi_{T}$. This fact together with the Remark 8.4 implies that the portion of the spectrum of $L \in U$ which lies in $\operatorname{int}(D)$ is not empty, call it $\Sigma(L)$. Define $X_{\Sigma(L)} \equiv \pi_{L} X$ and $T_{\Sigma(L)}=\left.T\right|_{X_{\Sigma(L)}}$. It is well know [28, p. 98] that $\operatorname{spec}\left(T_{\Sigma(L)}\right)=\Sigma(L)$. If $\Sigma(L)$ is not an unitary set, then $X_{\Sigma(L)}$ is not a uni-dimensional subspace and therefore $1 \neq \operatorname{dim}\left(X_{\Sigma(L)}\right)=\operatorname{dim}\left(\pi_{T} X\right)=1$. This contradiction shows that there is a unique simple eigenvalue $\lambda(L)$ of $L$ inside $D$.

By using the two previous claims one have a well defined mapping $U \ni L \mapsto \lambda(L) \in \mathbb{C}$, where $\lambda(L)$ is the unique simple eigenvalue of $L$ inside $\operatorname{int}(D)$. Now we proceed to the proof that this mapping is analytic. Fix $v \in X$ such that $\pi_{L} v$ is a non zero vector and choose $w \in X^{*}$ such that $w\left(\pi_{L} v\right) \equiv\left\langle w, \pi_{L} v\right\rangle \neq 0$ (Hahn Banach) for all $L$ in a small enough neighborhood of $T$. According to the Proposition 8.5 the operator $L$ commutes with $\pi_{L}$ then we get that $\left\langle w, \pi_{L}(L v)\right\rangle=\left\langle w, L \pi_{L}(v)\right\rangle=\lambda(L)\left\langle w, \pi_{L}(v)\right\rangle$ and consequently

$$
\lambda(L)=\frac{\left\langle w, \pi_{L}(L v)\right\rangle}{\left\langle w, \pi_{L}(v)\right\rangle} .
$$

From the Definition 8.3 and the above equality we obtain the analyticity of the mapping $U \ni L \mapsto \lambda(L) \in \mathbb{C}$.

\section{Spectral Gap and Exponential Decay}

In this section we will follow closely [2].

Definition 6.1. Consider the probability space $(\Omega, \mathcal{F}, \nu)$. Let $\sigma$ be the left shift on $\Omega$. For each $\varphi_{1}$ and $\varphi_{2}$ in $L^{2}(\Omega, \nu)$ we define the correlation function $C_{\varphi_{1}, \varphi_{2}, \nu}: \mathbb{Z} \rightarrow \mathbb{R}$ by

$$
C_{\varphi_{1}, \varphi_{2}, \nu}(n)=\int_{\Omega}\left(\varphi_{1} \circ \sigma^{n}\right) \varphi_{2} d \nu-\int_{\Omega} \varphi_{1} d \nu \int_{\Omega} \varphi_{2} d \nu
$$

Theorem 6.2. Suppose that $f \in W(\Omega)$ is a potential for which the Ruelle operator $\mathscr{L}_{f}$ has the spectral gap property. Consider the measure $\mu_{f}=h_{f} \nu_{f}$, where $\nu_{f}$ is the eigenmeasure given by the Theorem 4.4. Then the correlation function $C_{\varphi_{1}, \varphi_{2}, \mu_{f}}(n)$ decays exponentially fast. More precisely, there are $0<\widetilde{\tau}<1$ and $C(\widetilde{\tau})>0$ such that for all $\varphi_{1}, \varphi_{2} \in W(\Omega)$ the correlation function satisfies:

$$
\left|C_{\varphi_{1}, \varphi_{2}, \mu_{f}}(n)\right|=\left|\int_{\Omega}\left(\varphi_{1} \circ \sigma^{n}\right) \varphi_{2} d \mu_{f}-\int_{\Omega} \varphi_{1} d \mu_{f} \int_{\Omega} \varphi_{2} d \mu_{f}\right| \leq C_{1} \widetilde{\tau}^{n} .
$$

where $C_{1}=C(\widetilde{\tau})\left\|h_{f}\right\|_{0}\left(\int_{\Omega}\left|\varphi_{1}\right| d \nu_{f}\right)\left\|\varphi_{2}\right\|$.

Before prove the above theorem, we present two auxiliary lemmas.

Lemma 6.3. The spectral projection $\pi_{f} \equiv \pi_{\mathscr{L}_{f}}$ is given by $\pi_{f}(\varphi)=\left(\int_{\Omega} \varphi d \nu_{f}\right) \cdot h_{f}$. 
Proof. We know that $\pi_{f}$ and $\mathscr{L}_{f}$ commutes. By the Ruelle Theorem (Theorem 4.4) we have that $\lim _{n \rightarrow \infty}\left(1 / \lambda^{n}\right) \mathscr{L}_{f}^{n} \varphi=h_{f} \int_{\Omega} \varphi d \nu_{f}$ uniformly. Since $\pi_{f}$ is bounded we get that

$$
\left\|\pi_{f}\left(\lambda^{-n} \mathscr{L}_{f}^{n} \varphi-h_{f} \int_{\Omega} \varphi d \nu_{f}\right)\right\|_{0} \leq\left\|\pi_{f}\right\|\left\|\lambda^{-n} \mathscr{L}_{f}^{n} \varphi-h_{f} \int_{\Omega} \varphi d \nu_{f}\right\|_{0} \rightarrow 0,
$$

when $n \rightarrow \infty$. Since $\left.\pi_{f}\left(\lambda^{-n} \mathscr{L}_{f}^{n} \varphi\right)\right)=\lambda^{-n} \mathscr{L}_{f}^{n} \pi_{f}(\varphi)=\lambda^{n} \lambda^{-n} \pi_{f}(\varphi)=\pi_{f}(\varphi)$ we get that

$$
\pi_{f}(\varphi)=\pi_{f}\left(h_{f} \int_{\Omega} \varphi d \nu_{f}\right)=\int_{\Omega} \varphi d \nu_{f} \cdot \pi_{f}\left(h_{f}\right)=\int_{\Omega} \varphi d \nu_{f} \cdot h_{f} .
$$

Lemma 6.4. Let be $\varphi_{1}, \varphi_{2} \in W(\Omega)$ then $\mathscr{L}_{f}^{n}\left(\varphi_{1} \circ \sigma^{n} \cdot \varphi_{2} \cdot h_{f}\right)=\varphi_{1} \mathscr{L}_{f}^{n}\left(\varphi_{2} h_{f}\right)$.

Proof. The proof is an easy calculation. Let $x \in \Omega$ and $\varphi \in W(\Omega)$ then we have that $\mathscr{L}_{f}^{n} \varphi(x)=$ $\int_{M^{n}} \varphi(\mathbf{a} x) e^{S_{n}(\mathbf{a} x)} d \mu(\mathbf{a})$. Since $\varphi_{1} \circ \sigma^{n}(\mathbf{a} x)=\varphi_{1}(x) \quad \forall \mathbf{a} x, \mathbf{a} \in M^{n}$ and $\forall x \in \Omega$ we get that $\mathscr{L}_{f}^{n}\left(\varphi_{1} \circ \sigma^{n} \varphi_{2} h_{f}\right)(x)=\int_{M^{n}} \varphi_{1} \circ \sigma^{n} \varphi_{2} h_{f}(\mathbf{a} x) e^{S_{n}(\mathbf{a} x)} d \mu(\mathbf{a})=\varphi_{1}(x) \int_{M^{n}}\left(\varphi_{2} h_{f}\right)(\mathbf{a} x) e^{S_{n}(\mathbf{a} x)} d \mu(\mathbf{a})$.

Proof of the Theorem 6.2. Since $\mu_{f}=h_{f} d \nu_{f}$ it follows from the definition of the correlation function that

$$
\left|C_{\varphi_{1}, \varphi_{2}, \mu_{f}}(n)\right|=\left|\int_{\Omega}\left(\varphi_{1} \circ \sigma^{n}\right) \varphi_{2} h_{f} d \nu_{f}-\int_{\Omega} \varphi_{1} h_{f} d \nu_{f} \int_{\Omega} \varphi_{2} h_{f} d \nu_{f}\right| .
$$

Notice that $\left(\mathscr{L}_{f}^{*}\right)^{n} \nu_{f}=\lambda_{f}^{n} \nu_{f}$ and therefore the rhs above is equal to

$$
\left|\int_{\Omega} \lambda_{f}^{-n} \mathscr{L}_{f}^{n}\left(\left(\varphi_{1} \circ \sigma^{n}\right) \varphi_{2} h_{f}\right) d \nu_{f}-\int_{\Omega} \varphi_{1} h_{f} d \nu_{f} \int_{\Omega} \varphi_{2} h_{f} d \nu_{f}\right|
$$

By using the Lemma 6.4 and performing simple algebraic computations we get

$$
\left|C_{\varphi_{1}, \varphi_{2}, \mu_{f}}(n)\right| \leq\left(\int_{\Omega}\left|\varphi_{1}\right| d \nu_{f}\right)\left\|\lambda_{f}^{-n} \mathscr{L}_{f}^{n}\left(\varphi_{2} h_{f}-h_{f} \int_{\Omega} \varphi_{2} h_{f} d \nu_{f}\right)\right\|_{0} .
$$

We are supposing that the spectrum of $\mathscr{L}_{f}: W(\Omega) \rightarrow W(\Omega)$ consists in a simple eigenvalue $\lambda_{f}>0$ and a subset of a disc of radius strictly smaller than $\lambda_{f}$. Set $\tau=\sup \{|z| ;|z|<1$ and $z$. $\left.\lambda_{f} \in \sigma\left(\mathscr{L}_{f}\right)\right\}$. The existence of the spectral gap guarantees that $\tau<1$. Let $\pi_{f}$ the spectral projection associated to eigenvalue $\lambda_{f}$, then by the Proposition 8.6, the spectral radius of the operator $\mathscr{L}_{f}\left(I-\pi_{f}\right)$ is exactly $\tau \cdot \lambda_{f}$. Since the commutator $\left[\mathscr{L}_{f}, \pi_{f}\right]=0$, we get $\forall n \in \mathbb{N}$ that $\left[\mathscr{L}_{f}\left(I-\pi_{f}\right)\right]^{n}=\mathscr{L}_{f}^{n}\left(I-\pi_{f}\right)$. From the spectral radius formula (23) it follows that for each choice of $\widetilde{\tau}>\tau$ there is $n_{0} \equiv n_{0}(\widetilde{\tau}) \in \mathbb{N}$ so that for all $n \geq n_{0}$ we have $\left\|\mathscr{L}_{f}^{n}\left(\varphi-\pi_{f} \varphi\right)\right\| \leq$ $\lambda_{f}^{n} \widetilde{\tau}^{n}\|\varphi\|, \forall \varphi \in W(\Omega)$. Therefore there is a constant $C(\widetilde{\tau})>0$ such that for every $n \geq 1$

$$
\left\|\mathscr{L}_{f}^{n}\left(\varphi-\pi_{f} \varphi\right)\right\| \leq C(\widetilde{\tau}) \lambda_{f}^{n} \widetilde{\tau}^{n}\|\varphi\| \quad \forall \varphi \in W(\Omega) .
$$

By using the Lemma 6.3 and the above upper bound in the inequality (12) we obtain

$$
\left|C_{\varphi_{1}, \varphi_{2}, \mu_{f}}(n)\right| \leq\left(\int_{\Omega}\left|\varphi_{1}\right| d \nu_{f}\right) C \widetilde{\tau}^{n}\left\|\varphi_{2} h_{f}\right\| \leq C(\widetilde{\tau})\left\|h_{f}\right\|_{0}\left(\int_{\Omega}\left|\varphi_{1}\right| d \nu_{f}\right)\left\|\varphi_{2}\right\| .
$$




\section{Absence of the Spectral Gap in the Walters Space}

In this section we present the so-called long-range Ising model on the lattice $\mathbb{N}$ in the Thermodynamic Formalism setting. The goal is to exhibit explicitly a potential in the Walters space for which the associated Ruelle operator do not have the spectral gap.

Throughout this section we assume the metric space $(M, d)$ is given by $(\{-1,1\},|\cdot|)$, where $|\cdot|$ is the modulus function and the a priori probability measure $\nu=(1 / 2)\left[\delta_{\{-1\}}+\delta_{\{1\}}\right]$. Fix $\alpha>1$ and consider the potential $f: \Omega \rightarrow \mathbb{R}$ given by

$$
f(x)=-\sum_{n \geq 2} \frac{x_{1} x_{n}}{n^{\alpha}} .
$$

This potential is not $\gamma$-Hölder continuous for any $0<\gamma \leq 1$, see [9]. When $1<\alpha<2$, Dyson [12] proved that this model has spontaneous magnetization for sufficiently low temperatures. This fact for these models implies non-uniqueness DLR-Gibbs measures at such temperatures and also that the pressure can not be Fréchet-differentiable on a suitable Banach space. For $\alpha=2$ this phase transition result was proved by Frölich and Spencer [16]. On the other hand, when $\alpha>2$ the potential $f$ is in the Walters class. Indeed, for any choice of $n, p \in \mathbb{N}$ we have $\operatorname{var}_{n+p}\left(f(x)+f(\sigma(x))+\ldots+f\left(\sigma^{n-1}(x)\right)\right)=(n+p)^{-\alpha+1}+(n+p-1)^{-\alpha+1}+\ldots+p^{-\alpha+1}$ which implies that

$$
\sup _{n \in \mathbb{N}}\left[\operatorname{var}_{n+p}\left(f(x)+f(\sigma(x))+\ldots+f\left(\sigma^{n-1}(x)\right)\right)\right] \sim \sum_{j=p}^{\infty} j^{-\alpha+1} \sim p^{-\alpha+2}
$$

and then the Walters condition. For this reason, in what follows we assume that $\alpha>2$. In this case as mentioned in the introduction the potential $f$ belongs to an infinite dimensional subspace of $C(\Omega)$ as defined in [8] where the pressure is Fréchet-analytic. Note that the previous computation implies that this space can not be contained in the Hölder space.

In the Statistical Mechanics setting the potential $f$ is normally replaced/constructed by the absolutely uniformly summable interaction $\Phi=\left(\Phi_{A}\right)_{A \Subset \mathbb{N}}$, given by

$$
\Phi_{A}(x)= \begin{cases}\frac{x_{n} x_{m}}{|n-m|^{\alpha}}, & \text { if } A=\{n, m\} \subset \mathbb{N} \text { and } m \neq n \\ 0, & \text { otherwise }\end{cases}
$$

The relationship between the potential $f$ and the interaction $\Phi$ is detailed described in [9] and expressed by the following equality

$$
H_{n}(x)=\sum_{\substack{A \in \mathbb{N} \\ A \cap \Lambda_{n} \neq \emptyset}} \Phi_{A}(x)=f(x)+f(\sigma x)+\ldots+f\left(\sigma^{n-1} x\right) .
$$

Following [9, 21, 35] we construct a DLR-Gibbs measure as follows. Fix a potential $f$, and a boundary condition, which here for convenience will be chosen as $y=(1,1, \ldots) \in \Omega$. Now we take any cluster point (with respect to the weak topology) of the sequence $\left(\nu_{n}^{y}\right)_{n \in \mathbb{N}}$ in $\mathscr{P}(\Omega, \mathcal{F})$, 
where $\nu_{n}^{y}: \mathcal{F} \rightarrow[0,1]$ is the probability measure defined for each $F \in \mathcal{F}$ by the following expression:

$$
\nu_{n}^{y}(F)=\frac{1}{Z_{n}^{y}} \sum_{\substack{x \in \Omega ; \\ \sigma^{n}(x)=\sigma^{n}(y)}} 1_{F}(x) \exp \left(H_{n}(x)\right), \quad \text { where } \quad Z_{n}^{y}=\sum_{\substack{x \in \Omega ; \\ \sigma^{n}(x)=\sigma^{n}(y)}} \exp \left(H_{n}(x)\right) .
$$

Since $\alpha>2$ it is well-known that the sequence $\left(\nu_{n}^{y}\right)_{n \in \mathbb{N}}$ has a unique cluster point which will be denoted by $\nu_{\mathbb{N}}$. A proof of this classical fact, with a dynamical system point of view, using a consequence of the Dobrushin uniqueness theorem and also the Ruelle operator formalism is presented in [9].

Our next step is to construct a probability measure $\nu_{\mathbb{Z}}$ on the symbolic space $\hat{\Omega} \equiv\{-1,1\}^{\mathbb{Z}} \equiv$ $\{-1,1\}^{\mathbb{Z} \cap(-\infty, 0]} \times\{-1,1\}^{\mathbb{N}}$ such that

$$
\nu_{\mathbb{N}}(F)=\nu_{\mathbb{Z}}\left(\{-1,1\}^{\mathbb{Z} \cap(-\infty, 0]} \times F\right), \quad \forall F \in \mathcal{F} .
$$

Let us denote $\operatorname{Diag}(\mathbb{Z} \times \mathbb{Z}) \equiv\{(r, r): r \in \mathbb{Z}\}$ and $\mathbb{M} \equiv \mathbb{Z} \times \mathbb{Z} \backslash \operatorname{Diag}(\mathbb{Z} \times \mathbb{Z})$. We define a linear space $\mathbb{J} \subset \mathbb{R}^{\mathbb{M}} \equiv\left\{J_{i j} \in \mathbb{R}:(i, j) \in \mathbb{M}\right\}$ as being the set of points in $\mathbb{R}^{\mathbb{M}}$ satisfying $\sup _{i \in \mathbb{Z}} \sum_{j \in \mathbb{Z}: j \neq i}\left|J_{i j}\right|<\infty$. Let $J^{\Phi}$ and $T^{\Phi}$ be two points in $\mathbb{J}$ defined by $\left(J^{\Phi}\right)_{i j}=|i-j|^{-\alpha}$ for all $(i, j) \in \mathbb{M}$ and $\left(T^{\Phi}\right)_{i j}=\left(J^{\Phi}\right)_{i j}$ if $i, j \in \mathbb{N}$ with $i \neq j$ and $\left(T^{\Phi}\right)_{i j}=0$ otherwise. For each $n \in \mathbb{N}$ and $J \in \mathbb{J}$ we define the function $\mathscr{H}_{n}: \hat{\Omega} \times \mathbb{J} \rightarrow \mathbb{R}$ by

$$
\mathscr{H}_{n}(z, J)=\sum_{i=-n}^{n} \sum_{j \in \mathbb{Z}: j \neq i} J_{i j} z_{i} z_{j}
$$

For any fixed $J \in \mathbb{J}$ and $\hat{y}=(\ldots, 1,1,1, \ldots) \in \hat{\Omega}$ we can define, similarly as above, a probability measure $\nu_{n}^{\hat{y}, J}$ such that for each borelian $\hat{F}$ of $\hat{\Omega}$ we have

$$
\nu_{n}^{\hat{y}, J}(\hat{F})=\frac{1}{Z_{n}^{\hat{y}, J}} \sum_{\substack{z \in \hat{\Omega} ; z_{i}=1 \\ \forall i \in \mathbb{Z} \backslash\{-n, \ldots, n\}}} 1_{\hat{F}}(z) \exp \left(\mathscr{H}_{n}(z, J)\right), \quad \text { where } \quad Z_{n}^{\hat{y}, J}=\sum_{\substack{z \in \hat{\Omega} ; z_{i}=1 \\ \forall i \in \mathbb{Z} \backslash\{-n, \ldots, n\}}} \exp \left(\mathscr{H}_{n}(z, J)\right) .
$$

By straightforward computation we obtain, for each $n \in \mathbb{N}$ and $z \in \hat{\Omega}$ fixed, the following identities:

1. $\mathscr{H}_{n}\left(z, T^{\Phi}\right)=H_{n}\left(z_{1}, z_{2}, \ldots\right)$;

2. $Z_{n}^{\hat{y}, T^{\Phi}}=2^{n+1} Z_{n}^{y}$;

3. $1_{\left\{\{-1,1\}^{\mathbb{Z} \cap(-\infty, 0] \times F\}}\right.}(z)=1_{F}\left(z_{1}, z_{2}, \ldots\right)$.

Using the above three identities one can immediately see that

$$
\begin{aligned}
\nu_{n}^{\hat{y}, T^{\Phi}}\left(\left\{\{-1,1\}^{\mathbb{Z} \cap(-\infty, 0]} \times F\right)\right. & =\frac{1}{2^{n+1} Z_{n}^{y}} \sum_{\substack{z \in \hat{\Omega} ; z_{i}=1 \\
\forall i \in \mathbb{Z} \backslash\{-n, \ldots, n\}}} 1_{F}\left(z_{1}, z_{2}, \ldots\right) \exp \left(-H_{n}\left(z_{1}, z_{2}, \ldots\right)\right. \\
& =\nu_{n}^{y}(F) .
\end{aligned}
$$


Recalling that $\alpha>2$, it follows from classical results of the theory of DLR-Gibbs measures that the sequence $\left(\nu_{n}^{\hat{y}, J^{\Phi}}\right)_{n \in \mathbb{N}}$ has a unique cluster point which we call $\nu_{\mathbb{Z}}$. From the previous equality it easy to conclude that (14) is valid.

According to the definition of $\nu_{n}^{\hat{y}, J}$, for any fixed measurable set $\hat{F}$ and $n \in \mathbb{N}$ the function $\mathbb{J} \ni J \mapsto \nu_{n}^{\hat{y}, J}(\hat{F})$ is Fréchet-analytic, since it is just a finite sum of analytic functions. A straightforward computation shows that for each fixed $(i, j) \in \mathbb{M}$ we have

$$
\frac{\partial}{\partial J_{i j}} Z_{n}^{\hat{y}, J}=\sum_{\substack{z \in \hat{\Omega} ; z_{i}=1 \\ \forall i \in \mathbb{Z} \backslash\{-n, \ldots, n\}}} \frac{\partial}{\partial J_{i j}}\left(\mathscr{H}_{n}(z, J)\right) \cdot \exp \left(\mathscr{H}_{n}(z, J)\right)=\sum_{\substack{z \in \hat{\Omega} ; z_{i}=1 \\ \forall i \in \mathbb{Z} \backslash\{-n, \ldots, n\}}} z_{i} z_{j} \cdot \exp \left(\mathscr{H}_{n}(z, J)\right) .
$$

By multiplying and dividing the rhs by $Z_{n}^{\hat{y}, J}$ and use the definition of the Lebesgue integral we get

$$
\frac{\partial}{\partial J_{i j}} Z_{n}^{\hat{y}, J}=Z_{n}^{\hat{y}, J} \int_{\hat{\Omega}} z_{i} z_{j} d \nu_{n}^{y, J}(z)
$$

Performing similar computations and using the quotient rule, we have for any measurable function $\varphi: \hat{\Omega} \rightarrow \mathbb{R}$

$$
\begin{aligned}
\frac{\partial}{\partial J_{i j}} \nu_{n}^{\hat{y}, J}(\varphi) & =\frac{\partial}{\partial J_{i j}}\left[\frac{1}{Z_{n}^{\hat{y}, J}} \sum_{\substack{z \in \hat{\Omega} ; z_{i}=1 \\
\forall i \in \mathbb{Z} \backslash\{-n, \ldots, n\}}} \varphi(z) \exp \left(\mathscr{H}_{n}(z, J)\right)\right] \\
& =\int_{\hat{\Omega}} \varphi(z) z_{i} z_{j} d \nu_{n}^{y, J}(z)-\int_{\hat{\Omega}} \varphi(z) d \nu_{n}^{y, J}(z) \int_{\hat{\Omega}} z_{i} z_{j} d \nu_{n}^{y, J}(z) .
\end{aligned}
$$

Before proceed we state the GKS-II inequality but only in the generality required in this section. For more general cases, see [13, 25, 26, 23].

Theorem 7.1 (GKS-II Inequality [25, 26]). Fix $n \in \mathbb{N}$ and $\left\{n_{1}, n_{2}, \ldots, n_{k}\right\}$ an arbitrary subset of $\{-n, \ldots, n\}$. If $J \in \mathbb{J}$ satisfies $J_{i j} \geqslant 0$ for all $(i, j) \in \mathbb{M}$, then

$$
\int_{\hat{\Omega}} z_{n_{1}} \cdot \ldots \cdot z_{n_{k}} \cdot z_{i} \cdot z_{j} d \nu_{n}^{y, J}(z)-\int_{\hat{\Omega}} z_{n_{1}} \cdot \ldots \cdot z_{n_{k}} d \nu_{n}^{y, J}(z) \int_{\hat{\Omega}} z_{i} \cdot z_{j} d \nu_{n}^{y, J}(z) \geqslant 0,
$$

where $\nu_{n}^{y, J}$ denotes the probability measure defined in (16).

From now on we take $\varphi(z)=z_{1}$. Strictly speaking $\varphi$ is defined on $\hat{\Omega}$ but we will abuse notation and also use $\varphi(x)$ to denote the projection on the first coordinate of an element in $\Omega$. If $\widetilde{J} \in \mathbb{J}$ is such that $\widetilde{J}_{i j} \geqslant 0$ for all $(i, j) \in \mathbb{M}$ it follows from (18) and GKS-II inequality that

$$
\left.\frac{\partial}{\partial J_{i j}} \nu_{n}^{\hat{y}, J}(\varphi)\right|_{J=\widetilde{J}} \geqslant 0
$$

This inequality implies that the mapping $\mathbb{J} \ni J \mapsto \nu_{n}^{\hat{y}, J}(\varphi)$ is coordinatewise non-decreasing in $\mathbb{J} \cap[0,+\infty)^{\mathbb{M}}$. This monotonicity together with the inequalities $\left(T^{\Phi}\right)_{i j} \leqslant\left(J^{\Phi}\right)_{i j}$ immediately implies that

$$
\int_{\hat{\Omega}} \varphi(z) d \nu_{n}^{y, T^{\Phi}}(z) \leqslant \int_{\hat{\Omega}} \varphi(z) d \nu_{n}^{y, J^{\Phi}}(z)
$$


Since $\varphi$ is a simple function taking only the values -1 and 1 the lhs above is, from definition of the Lebesgue integral and the identity (17), equals to

$$
\begin{aligned}
\int_{\hat{\Omega}} \varphi(z) d \nu_{n}^{y, T^{\Phi}}(z) & =\nu_{n}^{y, T^{\Phi}}\left(\left\{z \in \hat{\Omega}: z_{1}=1\right\}\right)-\nu_{n}^{y, T^{\Phi}}\left(z \in \hat{\Omega}: z_{1}=-1\right) \\
& =\nu_{n}^{y}\left(\left\{x \in \Omega: x_{1}=1\right\}\right)-\nu_{n}^{y}\left(x \in \Omega: x_{1}=-1\right) \\
& =\int_{\Omega} \varphi(x) d \nu_{n}^{y}(x) .
\end{aligned}
$$

Replacing this last expression in the above inequality we arrive at

$$
\int_{\Omega} \varphi(x) d \nu_{n}^{y}(x) \leqslant \int_{\hat{\Omega}} \varphi(z) d \nu_{n}^{y, J^{\Phi}}(z)
$$

Since $\nu_{n}^{y, J^{\Phi}} \rightarrow \nu_{\mathbb{Z}}$ and $\nu_{n}^{y} \rightarrow \nu_{\mathbb{N}}$ it follows from the definition of weak convergence that

$$
\int_{\Omega} \varphi(x) d \nu_{\mathbb{N}}(x) \leqslant \int_{\hat{\Omega}} \varphi(z) d \nu_{\mathbb{Z}}(z) .
$$

To prove that the lhs above is non-negative we will use the GKS-I inequality. Here we also state it in the needed particular case. Its proof as well as its more general version can be found in $[13,24,23]$.

Theorem 7.2 (GKS-I Inequality [24]). Fix a natural number $n \geq 1$ and subset $\left\{n_{1}, n_{2}, \ldots, n_{k}\right\} \subset$ $\{-n, \ldots, n\}$ and $J \in \mathbb{J}$ satisfying $J_{i j} \geqslant 0$ for all $(i, j) \in \mathbb{M}$. If $\nu_{n}^{y, J}$ denotes the probability measure defined in (16), then

$$
\int_{\hat{\Omega}} z_{n_{1}} \cdot \ldots \cdot z_{n_{k}} \cdot z_{i} \cdot z_{j} d \nu_{n}^{y, J}(z) \geqslant 0
$$

By applying GKS-I inequality to the lhs of (19) and then taking the weak limit, when $n \rightarrow \infty$ we get

$$
0 \leqslant \int_{\Omega} \varphi(x) d \nu_{\mathbb{N}}(x) \leqslant \int_{\hat{\Omega}} \varphi(z) d \nu_{\mathbb{Z}}(z)
$$

Since $\alpha>2$, there is a theorem ensuring that $\int_{\hat{\Omega}} \varphi d \nu_{\mathbb{Z}}(z)=0$, see [13]. Therefore we have proved that

$$
\int_{\Omega} \varphi(x) d \nu_{\mathbb{N}}(x)=0
$$

To get the lower bound we are interested we define for $n \geq 1$ the element $J^{\{1, n+1\}} \in \mathbb{J}$, where $\left(J^{\{1, n+1\}}\right)_{i j}=n^{-\alpha}$ if $(i, j)=(1, n+1)$ and $\left(J^{\{1, n+1\}}\right)_{i j}=0$ otherwise. Similarly as above, we obtain by another application of the GKS-II inequality the coordinatewise monotonicity of the mapping $\mathbb{J} \ni J \mapsto \nu_{m}^{\hat{y}, J}\left(z_{n+1} z_{1}\right)$, where $m>n$, therefore we can conclude that

$$
\int_{\hat{\Omega}} z_{n+1} z_{1} d \nu_{m}^{y, J^{\{1, n+1\}}}(z) \leqslant \int_{\hat{\Omega}} z_{n+1} z_{1} d \nu_{m}^{y, J^{\Phi}}(z) .
$$


Notice that the lhs above can be explicitly computed as follows (and its value is independent of $m$ )

$$
\begin{aligned}
\int_{\hat{\Omega}} z_{n+1} z_{1} d \nu_{m}^{y, J^{\{1, n+1\}}}(z) & =\sum_{z_{n+1}= \pm 1} \sum_{z_{1}= \pm 1} z_{n+1} z_{1} \exp \left(\frac{z_{n+1} z_{1}}{n^{\alpha}}\right)\left[\sum_{z_{n+1}= \pm 1} \sum_{z_{1}= \pm 1} \exp \left(\frac{z_{n+1} z_{1}}{n^{\alpha}}\right)\right]^{-1} \\
& =\frac{2 \exp \left(n^{-\alpha}\right)-2 \exp \left(n^{-\alpha}\right)}{2 \exp \left(n^{-\alpha}\right)+2 \exp \left(n^{-\alpha}\right)} \\
& =\tanh \left(n^{-\alpha}\right) .
\end{aligned}
$$

On the other hand, by using the previous equality and (17) we get for any $m>n$

$$
\tanh \left(n^{-\alpha}\right) \leqslant \int_{\hat{\Omega}} z_{n+1} z_{1} d \nu_{m}^{y, J^{\Phi}}(z)=\int_{\Omega} x_{n+1} x_{1} d \nu_{m}^{y}(x)=\int_{\Omega}\left(\varphi \circ \sigma^{n}\right) \varphi d \nu_{m}^{y}(x) .
$$

By Taylor expanding the hyperbolic tangent and taking the weak limit when $m \rightarrow \infty$, we get the following inequality for some constant $C>0$

$$
\int_{\Omega}\left(\varphi \circ \sigma^{n}\right) \varphi d \nu_{\mathbb{N}} \geqslant \tanh \left(n^{-\alpha}\right) \geqslant \frac{C}{|n|^{\alpha}} .
$$

Piecing together the previous inequality and (20) we finally arrived at

$$
\frac{C}{|n|^{\alpha}} \leqslant \int_{\Omega}\left(\varphi \circ \sigma^{n}\right) \varphi d \nu_{\mathbb{N}}-\int_{\Omega} \varphi d \nu_{\mathbb{N}} \int_{\Omega} \varphi d \nu_{\mathbb{N}}=C_{\varphi, \varphi, \nu_{\mathbb{N}}}(n) .
$$

It was shown in [9] that $\mu_{f} \equiv h_{f} d \nu_{f}$, where $\nu_{f}$ and $h_{f}$ is given by Theorem 4.4, belongs to $\mathcal{G}^{D L R}(f)$. The authors also shown that for $\alpha>2$ the set $\mathcal{G}^{D L R}(f)$ is a singleton and therefore $\mu_{f}=\nu_{\mathbb{N}}$. This fact together with the continuity of $h_{f}$ and the previous inequality shows that $C_{\varphi, \varphi, \mu_{f}}(n)$ can not decays exponentially fast. Since $\varphi(x)=x_{1}$ is in the Walters class it follows from the Theorem 6.2 that $\mathscr{L}_{f}$ has not the spectral gap property.

\section{Appendix}

\section{Analyticity on Banach Spaces}

Definition 8.1. Let $\left(X,\|\cdot\|_{X}\right)$ and $\left(Y,\|\cdot\|_{Y}\right)$ be Banach spaces and $U$ an open subset of $X$. For each $k \in \mathbb{N}$, a function $F: U \rightarrow Y$ is said to be $k$-differentiable in $x$ if for $j \in\{1, \ldots, k\}$, there exist a j-linear bounded transformation $D^{j} F(x): X^{j} \rightarrow Y$ such that

$$
D^{j-1} F\left(x+v_{j}\right)\left(v_{1}, \ldots, v_{j-1}\right)-D^{j-1} F(x)\left(v_{1}, \ldots, v_{j-1}\right)=D^{j} F(x)\left(v_{1}, \ldots, v_{j}\right)+o_{j}\left(v_{j}\right)
$$

where $o_{j}: X \rightarrow Y$ is such that $\lim _{v \rightarrow 0}\left\|o_{j}(v)\right\|_{Y} /\left(\|v\|_{X}\right)=0$.

We say that $F$ has derivatives of all orders in $U$, if for any $k \in \mathbb{N}$, and any $x \in U, F$ is $k$-differentiable in $x$. 
Definition 8.2. Let $X$ and $Y$ be Banach spaces and $U$ an open subset of $X$. A function $F: U \rightarrow Y$ is called analytic on $U$, when $F$ has derivatives of all orders in $U$, and for each $x \in U$ there exists an open neighborhood $U_{x}$ of $x$ in $U$ such that for all $v \in U_{x}$, we have that

$$
F(x+v)-F(x)=\sum_{j=1}^{\infty} \frac{1}{n !} D^{j} F(x) v^{j},
$$

where $D^{j} F(x) v^{j}=D^{j} F(x)(v, \ldots, v)$ and $D^{j} F(x)$ is the $j$-th derivative of $F$ in $x$.

If $F: U \rightarrow Y$ is analytic on $U$, then for each $n \in \mathbb{N}$, the Taylor expansion of order $n$ is

$$
F(x+v)=F(x)+D^{1} F(x) v+\frac{D^{2} F(x) v^{2}}{2}+\frac{D^{3} F(x) v^{3}}{6}+\ldots+\frac{D^{n} F(x) v^{n}}{n !}+o_{n+1}(v),
$$

where $o_{n+1}(v)=\sum_{j=n+1}^{\infty}(1 / n !) D^{j} F(x) v^{j}$ satisfies $\lim _{v \rightarrow 0}\left\|o_{n+1}(v)\right\|_{Y} /\|v\|_{X}^{n}=0$.

\section{Some background on spectral theory}

In this section we list some classical results of Spectral Theory for more details and proofs see [28]. Let $X$ be a Banach space and $T: X \rightarrow X$ a bounded operator, we define the spectrum of the operator $T$ by

$$
\operatorname{spec}(T)=\left\{\lambda \in \mathbb{C} ;(\lambda I-T)^{-1} \text { do not exists }\right\} .
$$

The resolvent set $\rho(T)$ of $T$ is defined as the complement of $\operatorname{spec}(T)$. The resolvent set of a bounded operator is an open set while the spectrum is a compact set. The spectral radius of the operator $T$ is defined as $r(T)=\sup \{|x-y| ; x, y \in \operatorname{spec}(T)\}$. The spectral radius has the following characterization

$$
r(T)=\liminf _{n}\left\|T^{n}\right\|^{\frac{1}{n}}=\lim _{n \rightarrow \infty}\left\|T^{n}\right\|^{\frac{1}{n}} .
$$

It is also known that $\operatorname{spec}(T) \subset B(0, r(T))$ and $\operatorname{spec}(T)=\operatorname{spec}\left(T^{*}\right)$, where $T^{*}: X^{*} \rightarrow X^{*}$ is the adjoint of $T$.

Definition 8.3. Let $T: X \rightarrow X$ be a bounded linear operator and $\gamma$ a rectifiable Jordan curve that lies in $\rho(T)$, then we define the spectral projection $\pi_{T}: X \rightarrow X$ as follows

$$
\pi_{T}=\frac{1}{2 \pi} \int_{\gamma}(\lambda I-T)^{-1} d \lambda .
$$

Remark 8.4. If the interior of $\gamma$ lies in the interior of $\rho(T)$ then $\pi_{T}=0$. On the other hand if $\operatorname{spec}(T)$ lies entirely in the interior of $\gamma$ then $\pi_{L}=I d$.

Proposition 8.5. If $T: X \rightarrow X$ is bounded then $\pi_{T}$ is a projection, i.e, $\pi_{T}^{2}=\pi_{T}$. Moreover $\pi_{T}$ commutes with $T$ 
A subset of $\operatorname{spec}(T)$ which is both open and closed in $\operatorname{spec}(T)$ is called a spectral set. Let $\Sigma(T) \subset \operatorname{spec}(T)$ be a spectral set, and $\gamma$ a rectifiable Jordan curve which lies in $\rho(T)$ containing $\Sigma(T)$ in its interior. Denote by $\pi_{T, \Sigma(T)}$ the spectral projection associated with $T$ and $\gamma$, i.e.,

$$
\pi_{T, \Sigma(T)}=\frac{1}{2 \pi} \int_{\gamma}(\lambda I-T)^{-1} d \lambda,
$$

where $\gamma$ is any rectifiable Jordan curve surrounding the spectral set $\Sigma(T)$, completely contained in the $\rho(T)$ and such that any other point in the spectrum is outside $\gamma$.

We use the notation $X_{\Sigma(T)}=\pi_{T, \Sigma(T)} X$ and $T_{\Sigma(T)}=\left.T\right|_{X_{\Sigma(T)}}$.

Proposition 8.6. Let be $\Sigma(T)$ a spectral set of $\operatorname{spec}(T)$ then $\operatorname{spec}\left(T_{\Sigma(T)}\right)=\Sigma(T)$.

Proposition 8.7. Let $\pi_{1}, \pi_{2}: X \rightarrow X$ be linear projections, then there exists $\epsilon>0$ such that if $\left\|\pi_{1}-\pi_{2}\right\|<\epsilon$ then $\pi_{1}$ and $\pi_{2}$ has the same rank, i.e, $\operatorname{dim} \pi_{1}(X)=\operatorname{dim} \pi_{2}(X)$.

\section{Acknowledgments}

We thank Aernout van Enter for calling to our attention the references [8, 10]. We also thank Artur Lopes and Rodrigo Bissacot for their suggestions and comments on the early version of this manuscript. Leandro Cioletti is partially supported by FEMAT and Eduardo Silva is supported by CNPq.

\section{References}

[1] A. T. Baraviera, L. M. Cioletti, A. O. Lopes, J. Mohr and R. R. Souza. On the general one-dimensional XY Model: positive and zero temperature, selection and non-selection. Rev. Math. Phys., v. 23, p. 1063-1113, 2011.

[2] V. Baladi. Positive Transfer Operators and Decay of Correlations. World Scientific Publishing Co., 2000.

[3] L. BARreira . Thermodynamic Formalism and Applications to Dimension Theory. Birkhauser, 2010.

[4] R. Bissacot, E. Garibaldi. Weak KAM methods and ergodic optimal problems for countable Markov shifts. Bull. Braz. Math. Soc., v. 41, p. 321-338, 2010.

[5] T. Bousch. La condition de Walters. Ann. Sci. École Norm. Sup., v. 34 p. 287-311, 2001.

[6] R. Bowen. Equilibrium states and the ergodic theory of Anosov diffeomorphisms. Lecture Notes in Mathematics, v. 470, Springer, 1994.

[7] R. Bowen. Hausdorff dimensions of quasicircles. IHES Publ. Math., v. 50, p. 11-25, 1977. 
[8] M. Cassandro And E. Olivieri. Renormalization group and analyticity in one dimension: a proof of Dobrushin's theorem, Comm. Math. Phys., v. 80, p. 255-269, 1981.

[9] L. Cioletti And A. Lopes. Interactions, Specifications, DLR probabilities and the Ruelle Operator in the One-Dimensional Lattice. ArXiv-1404.3232, 2014.

[10] R. L. Dobrushin. Analyticity of correlation functions in one-dimensional classical systems with slowly decreasing potentials. Comm. Math. Phys., v. 32, p. 269-289, 1973.

[11] Y. Daon. Bernoullicity of equilibrium measures on countable Markov shifts Discrete Contin. Dyn. Syst., v. 33, p. 4003-4015, 2013.

[12] F. J. Dyson. Existence of a phase-transition in a one-dimensional Ising ferromagnet. Comm. Math. Phys., v. 12, p. 91-107, 1969.

[13] R. ElLis Entropy, Large Deviation and Statistical Mechanics. Springer, 2005.

[14] A.H. FAn. A proof of the Ruelle theorem. Reviews Math. Phys., v. 7, p. 1241-1247, 1995.

[15] A. Fan, Y. Jiang. On Ruelle-Perron-Frobenius Operators. I. Ruelle Theorem. Comm. Math. Phys., v. 223, p. 125-141, 2001.

[16] J. FrÖHLich, T. SPENCER. The phase transition in the one-dimensional Ising model with $1 / r^{2}$ interaction energy. Comm. Math. Phys. v. 84, p. 87-101, 1982.

[17] J, Glimm And A. JAFFe. Quantum physics: A functional integral point of view. Second edition, Springer-Verlag, New York, 1987.

[18] O. Jenkinson. Ergodic optimization, Discrete and Continuous. Dynamical Systems, Series A, v. 15, p. 197-224, 2006.

[19] O. Jenkinson, D. Mauldin and M. URBanski. Ergodic optimization for countable alphabet subshifts of finite type. Erg.Theo. and Dyn. Syst., v. 26, p. 1791-1803, 2006.

[20] Y. P. JiAng. A Proof of existence and simplicity of a maximal eigenvalue for RuellePerron-Frobenius operators. Letters in Math. Phys., v. 48, p. 211-219, 1999.

[21] H.-O. Georgir, Gibbs Measures and Phase Transitions. de Gruyter, Berlin, 1988.

[22] S. GouËzEL. Sharp polynomial estimates for the decay of correlations. Israel J. Math., v. 139, p. 29-65, 2004.

[23] J. Ginibre. General formulation of Griffiths' inequalities. Comm. Math. Phys., v. 16, p. 310-328, 1970.

[24] R. B. Griffiths. Correlations in Ising Ferromagnets. I. J. Math. Phys. v. 8, p. 478-483, 1967. 
[25] R. B. GRIfFiths. Rigorous results for Ising ferromagnets of arbitrary spin. J. Math. Phys., v. 10, p. 1559-1565, 1969.

[26] D. G. Kelly and S. Sherman. General Griffiths' Inequalities on Correlations in Ising Ferromagnets. J. Math. Phys. v. 9, p. 466-484, 1968.

[27] A. O. Lopes, J. Mohr, J. Mengue, And R. R. Souza. Entropy and Variational Principle for one-dimensional Lattice Systems with a general a-priori measure: finite and zero temperature. Erg. Theo. and Dyn. Syst., v. 35, p. 1925-1961, 2015.

[28] E. Lorch. Spectral theory. University Texts in the Mathematical Sciences, Oxford University Press, New York, 1962.

[29] R. MAÑé. Generic properties and problems of minimizing measures of Lagrangian systems. Nonlinearity, v. 9, p. 273-310, 1996.

[30] R. MAÑÉ. The Hausdorff dimension of horseshoes of diffeomorphisms of surfaces, Bol. Soc. Brasil. Mat., v. 20, p. 1-24, 1990.

[31] A. Manning, H. McCluskey. Hausdorff dimension for horseshoes, Erg. Theo. and Dyn. Syst., v. 3, p. 251-260, 1983.

[32] W. Parry And M. PollicotT. Zeta functions and the periodic orbit structure of hyperbolic dynamics. Astérisque, v. 187-188, 1990.

[33] Y. B. PEsin. Dimension theory in dynamical systems contemporary views and application. Chicago Lectures in Mathematics Series, 1997.

[34] D. Ruelle. Statistical mechanics of a one-dimensional lattice gas. Comm. Math. Phys. v. 9, p. 267-278, 1968.

[35] D. RuelLE. Thermodynamic Formalism. Second edition, Cambridge, 2004.

[36] O. SARIG . Thermodynamic formalism for countable markov shifts. Erg. Theo. and Dyn. Syst., v. 19, p. 1565-1593, 1999.

[37] O. SARIG. Subexponential decay of correlations. Invent. Math., v. 150, p. 629-653, 2002.

[38] O. SARIG . Lecture Notes on Thermodynamic Formalism for Topological Markov Shifts. Preprint PenState USA, 2009.

[39] E. A. Silva, R. R. Silva And R. R. Souza. The analyticity of a generalized Ruelle operator. Bull. Braz. Math. Soc., v. 45, p. 1-20, 2014.

[40] E. A. Silva. Dimensão de Hausdorff de Ferraduras. Universidade de Brasília Departamento de Matemática - Monografia de Mestrado, 2010.

[41] F. SpItzer. A Variational characterization of finite Markov chains. The Annals of Mathematical Statistics. v. 43, p. 303-307, 1972. 
[42] M. VianA. Stochastic dynamics of deterministic systems. Notes IMPA, v. 21, 1997.

[43] P. Walters. A natural space of functions for the Ruelle operator theorem. Erg. Theo. and Dyn. Syst., v. 27, p. 1323-1348, 2007.

[44] P. Walters. Invariant Measures and Equilibrium States for Some Mappings which Expand Distances. Trans. Amer. Math. Soc., v. 236, p. 121-153, 1978.

\author{
LEANDRo CioletTi \\ EduARdo ANTÔNIO DA Silva \\ Departamento de Matemática \\ UNIVERSIDADE DE BRASÍLIA \\ Campus Universitário Darcy Ribeiro - Asa Norte \\ 70910-900 BRASÍLIA - DF - BRAZIL. \\ cioletti@mat.unb.br \\ eduardo23maf@gmail.com
}

